\title{
Suppression of Hopf bifurcation in metal cutting by extrusion machining
}

\author{
Yao Liu · Songlin Cai · Xinchun Shang • \\ Lanhong Dai
}

Received: 29 April 2016/ Accepted: 28 November 2016 / Published online: 9 December 2016

(C) Springer Science+Business Media Dordrecht 2016

\begin{abstract}
Hopf bifurcation is a common phenomenon during the metal cutting process, which results in poor surface finish of the workpiece and inhomogeneous grain structure in materials. Therefore, understanding and controlling Hopf bifurcation in metal cutting are necessary. In this work, the systematic low-speed extrusion machining experiments were conducted to suppress Hopf bifurcation phenomenon. It is found that the suppression of Hopf bifurcation is achieved with the increasing constraint extrusion degree. In order to reveal the mechanism of the suppression of Hopf bifurcation, a new nonlinear dynamic model for extrusion machining is developed where the convection, the diffusion, the extrusion of constraint, the thermal-softening deformation and the fracture-type damage are included. The theoretical predictions are in agreement with the experimental results; therefore,
\end{abstract}

Y. Liu $\cdot$ X. Shang

School of Mathematics and Physics, University of Science and Technology Beijing, Beijing 100083, People's Republic of China

S. Cai $(\varangle) \cdot$ L. Dai $(\varangle)$

State Key Laboratory of Nonlinear Mechanics, Institute of Mechanics, Chinese Academy of Sciences, Beijing 100190, People's Republic of China e-mail: caisonglin@lnm.imech.ac.cn

L. Dai

e-mail: lhdai@1nm.imech.ac.cn

S. Cai

China Electric Power Research Institute, State Grid Corporation of China, Beijing 100192, People's Republic of China the present theoretical model is effective to characterize the suppression of Hopf bifurcation in metal cutting. Based on the numerical calculation of the theoretical model, the mechanisms underlying in extrusion machining are further revealed: Fracture-type deformation is more important than the thermal-softeningtype deformation in low-speed extrusion machining; however, the thermal-softening-type deformation is the primary deformation mode for high-speed extrusion machining.

Keywords Hopf bifurcation - Metal cutting · Bifurcation suppression · Nonlinear dynamic model

\section{Introduction}

Metal cutting, simply machining, is a process of removing excess material from the workpiece in the form of chips [1]. Chips undergoing severe plastic deformation (SPD) during machining are composed of ultrafinegrained structures. Machining has been demonstrated to be a low-cost method for producing materials of high strength [2-4]. The serrated chips are more prone to appear during machining difficult-to-cut materials. For manufacturing, the occurrence of serrated chips leads to bad surface finish and increased tool wear [5]. For producing ultrafine-grained materials, the serrated chips result in inhomogeneous grain structure in materials [6]. Therefore, the serrated chips should be avoided. Extensive research has been devoted to understand- 
ing the mechanisms of serrated chip formation and the methods controlling serrated chips.

For the mechanism of serrated chips, there are two Hopf bifurcations occurring during the formation of serrated chips: the shear band type deformation where shear deformation within primary shear zone (PZS) is unstable and shear bands emerge in serrated chips, and the fracture-type deformation where microcracks form within PSZ and materials finally fracture in serrated chips. In terms of shear band type deformation, Recht [7] carried out a pioneering study on the transition from continuous to serrated chip formation with increasing cutting speed. In his opinion, the catastrophic thermoplastic shear bands will occur if the thermal softening in materials overcomes the strain hardening effects. Considering the simple heat-transfer analysis in metal cutting, Semiatin and Rao [8] proposed an analytical model to give the quantitative prediction of the critical speed for the serrated chip formation. Molinari and Dudzinski [9] considered a viscoplastic material model in machining and discussed the influence of material parameters on the heterogeneity of the plastic flow. Burns and Davies $[10,11]$ put forward a lumped-parameter model for chip segmentation in high-speed machining to explain the adiabatic shear localization-induced segmented chip as a supercritical Hopf bifurcation. Considering the effect of strain gradient in shear bands, Huang and Aifantis [12], Huang et al. [13] presented a method for thermo-viscoplastic instability to describe the serrated chip flow. Molinari et al. [14] investigated the role of cutting conditions on chip serration by combining finite element calculations and analytical modeling.

As for the oscillations of tool, another Hopf bifurcation appears, where a time-delayed system due to regenerative effect becomes unstable and leads to the occurrence of machine-tool chatter. Hanna and Tobias [15] presented the first mathematical model of nonlinear machine-tool chatter. Shi and Tobias [16] extended the work of Hanna and Tobias to analyze the process whereby the cutting tool leaves the surface of materials. Based on the model of Hanna and Tobias, Nayfeh et al. [17] used the method of multiple scales to research the behavior of the Hopf bifurcations in metal cutting. Pratt and Nayfeh [18] proposed a twodegrees-of-freedom system with time delay to examine the subcritical instabilities in boring bars. KalmárNagy et al. [19] put forward a nonlinear second- order ordinary differential equation with time delay to model a machine tool with regenerative effects. Recently, Nayfeh and Nayfeh [20] used a systematic approach to investigate the local and global dynamics of cutting tool on a lathe. Ren et al. [21] presented fuzzy acoustic emission identification in highprecision hard turning process. Moradi et al. [22] investigated the occurrence of various types of bifurcation in milling process with tool-wear and process damping effects. Litak et al. [23] examined the regenerative cutting process by using a single-degree-offreedom nonsmooth model with a friction component and a time delay term. Elias and Namboothiri [24] put forward a cross-recurrence plot methodology to recognize the transition from regular cutting to the chatter cutting. Molnár et al. [25] presented a statedependent distributed-delay model of orthogonal cutting to describe the dynamics of the tool-workpiece system. Otto and Radons [5] analyzed the influence of tangential and torsional vibrations on the stability lobes in metal cutting.

The aforementioned works focus on machining in manufacture where high-speed machining (HSM) is needed. It is demonstrated that serrated chips in HSM are treated as a Hopf bifurcation due to shear banding deformation $[10,26,27]$. Based on the results from the literature [4,28], temperature in machining increases with the increasing cutting speed and recrystallization happens when the cutting speed exceeds a certain value. Because recrystallization affects the grain refinement and resulting hardness in HSM [4], ultrafine-grained materials are produced during lowspeed machining (LSM) [29]. For LSM, fracture typically results in the formation of serrated chips [30]. Serrated chips are harmful for both manufacturing and production of ultrafine-grained materials; therefore, serrated chips need to be suppressed during machining. For the suppression of serrated chips in HSM, Cai and Dai [31] put forward a thermal-softening-based dynamic large strain extrusion machining model to explain the suppression of shear banding type serrated chips. However, the suppression of serrated chips in LSM is vacant and the mechanism on how fracture affects the Hopf bifurcation in LSM is unclear. In this paper, a low-speed extrusion machining device is used to research the suppression of serrated chips in LSM. To elucidate the effect of fracture and shear banding on Hopf bifurcation, a theoretical model for machining is estab- 
lished where convection, diffusion, extrusion of constraint, thermal-softening deformation and fracturetype damage are included. The analytical results are in agreement with the available experimental results, and the underlying physics during machining are clearly revealed by the numerical calculations of the presented model.

\section{Experimental procedure}

The sample materials used in the experiments are magnesium alloy AZ31B and aluminum alloy 7075-T6 with chemical composition specified in Table 1 . The annealing temperatures of AZ31B and 7075-T6 are 345 and $413^{\circ} \mathrm{C}$, respectively. The mechanical properties of AZ31B and 7075-T6 are listed in Table 2. The microstructures of three section planes in the sample of AZ31B before machining are shown in Fig. 1.

Figure 1 shows the process of low-speed extrusion machining which is conducted on material testing machine. The workpiece with a precut depth $t_{0}$ is moving toward the tool at the cutting speed $V_{0}$, and the chip thickness $t_{c}$ is controlled by the constraint. Figure 2 shows a schematic of extrusion machining, in which the free machining (FM) is marked by the lines OBC. The chip thickness in FM is labeled as $t_{c}^{*}$. Here, an orthogonal machining process is taken into consideration, where the wedge-shaped tool with a rake angle $\alpha$ is static, and the workpiece with a cutting layer depth $t_{0}$ is moving toward the tool. Finally, because of the extrusion from constraint, the workpiece materials in the cutting layer flow out along the rake face of the tool in the form of a chip with a thickness $t_{c}$.

Table 1 Chemical compositions of magnesium alloy AZ31B and aluminum alloy 7075-T6

\begin{tabular}{|c|c|c|c|c|c|c|c|c|}
\hline \multicolumn{9}{|l|}{ AZ31B } \\
\hline Elements & $\mathrm{Mg}$ & $\mathrm{Al}$ & $\mathrm{Zn}$ & $\mathrm{Mn}$ & $\mathrm{Si}$ & $\mathrm{Cu}$ & $\mathrm{Ca}$ & Others \\
\hline $\mathrm{Wt}(\%)$ & 97 & $2.5-3.5$ & $0.6-1.4$ & 0.2 & 0.1 & 0.05 & 0.04 & $\leq 0.01$ \\
\hline \multicolumn{9}{|l|}{ 7075-T6 } \\
\hline Elements & $\mathrm{Al}$ & $\mathrm{Zn}$ & $\mathrm{Mg}$ & $\mathrm{Cu}$ & $\mathrm{Cr}$ & $\mathrm{Fe}$ & $\mathrm{Si}$ & Others \\
\hline Wt (\%) & 89.3 & 5.6 & 2.5 & 1.6 & 0.23 & $\leq 0.5$ & $\leq 0.4$ & $\leq 0.7$ \\
\hline
\end{tabular}

Table 2 Mechanical properties for magnesium alloy AZ31B and aluminum alloy 7075-T6

\begin{tabular}{llll}
\hline Properties and parameters & Notation & AZ31B & $7075-\mathrm{T} 6$ \\
\hline Density & $\rho\left(\mathrm{kg} \mathrm{m}^{-3}\right)$ & 1770 & 2810 \\
Elastic modulus & $E(\mathrm{GPa})$ & 45 & 70 \\
Thermal conductivity & $k\left(\mathrm{w} \mathrm{m}^{-1} \mathrm{~K}^{-1}\right)$ & 96 & 130 \\
Specific heat capacity & $c\left(\mathrm{~J} \mathrm{~kg}^{-1} \mathrm{~K}^{-1}\right)$ & 1000 & 960 \\
Taylor-Quinney coefficient & $\beta$ & 0.9 & 0.9 \\
Contact length & $L_{2}(\mu \mathrm{m})$ & $\sim 5$ & $\sim 5$ \\
Shear yield stress & $\tau_{y}(\mathrm{MPa})$ & 90 & 290 \\
Strain rate dependency coefficient & $C$ & 0.2 & 0.34 \\
Thermal softening exponent & $m$ & 1 & 1 \\
Reference strain rate & $\dot{\bar{\gamma}}\left(\mathrm{s}^{-1}\right)$ & $\sqrt{3} \times 10^{-5}$ & $\sqrt{3} \times 10^{-5}$ \\
Ambient temperature & $T_{a}(\mathrm{~K})$ & 300 & 300 \\
Melting temperature & $T_{m}(\mathrm{~K})$ & 878 & 908 \\
Damage diffusion length & $\tilde{l}(\mu \mathrm{m})$ & 10 & 50 \\
Diffusion coefficient of the crystal defect & $\tilde{\eta}\left(\mathrm{m}^{2} / \mathrm{s}\right)$ & $1 \times 10^{-9}$ & $1 \times 10^{-9}$ \\
Fracture toughness & $K_{C}(\mathrm{MPa} \sqrt{m})$ & 17.16 & 45 \\
Poisson's ratio & $v$ & 0.35 & 0.33 \\
& & & \\
\hline
\end{tabular}




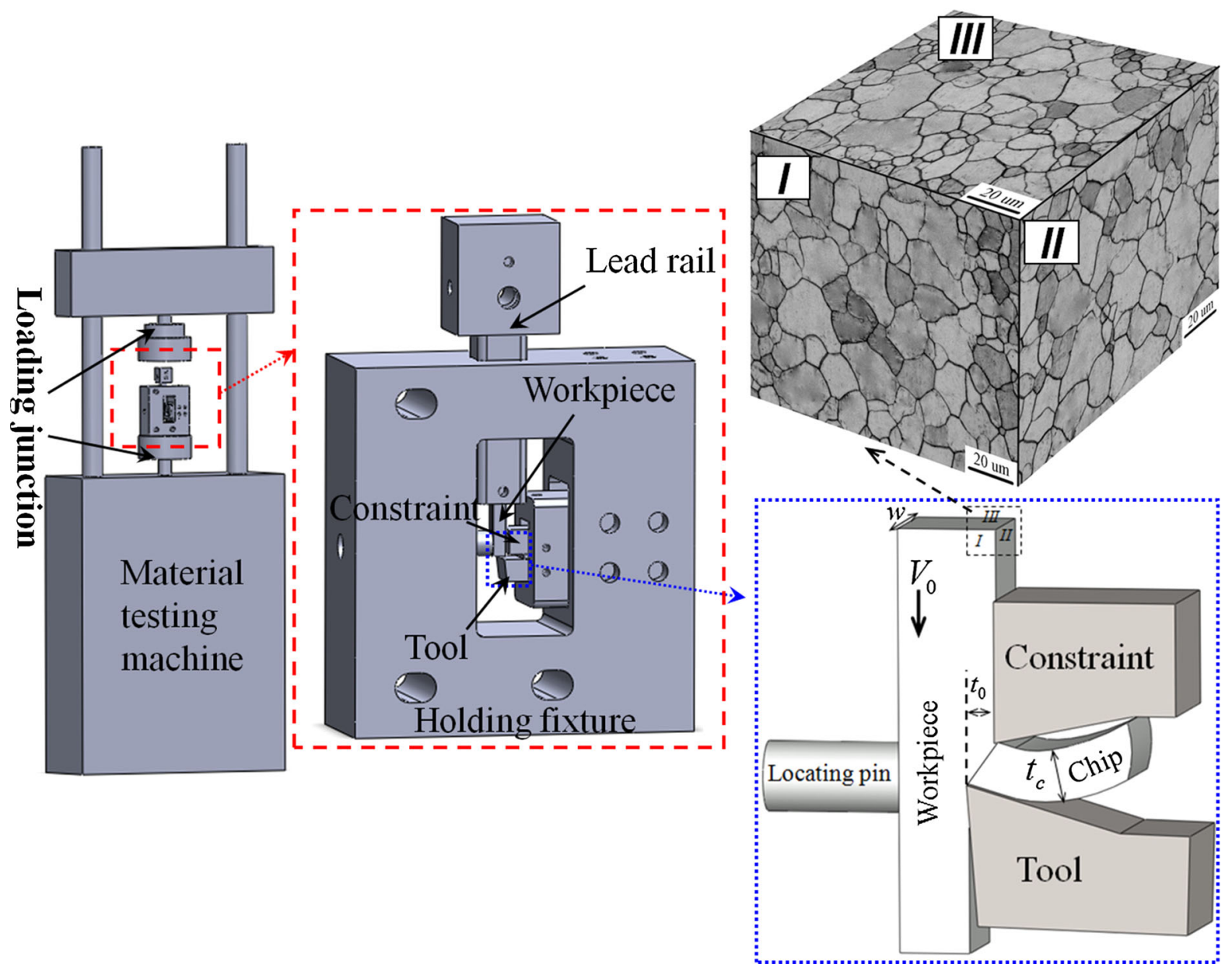

Fig. 1 Schematic of low-speed extrusion machining process and the microstructures of three section planes of workpiece before machining

The chip is formed by a process of shear which is approximately confined to a zone called primary shear zone (PSZ) OA. The inclined angle $\varphi$ of PSZ is named as shear angle. According to the geometrical relationship [32], the shear angle $\varphi$ is achieved by $\tan \varphi=$ $t_{0} \cos \alpha /\left(t_{c}-t_{0} \sin \alpha\right)$. Based on the definition of constraint extrusion factor $(\mathrm{CEF}) \chi=\left(t_{c}^{*}-t_{c}\right) / t_{c}^{*}(\chi \in$ $[0,1])[31]$, the different constraint extrusion effects can be obtained by changing the position of constraint in the experiments. In order to explore the relationship between different CEFs and Hopf bifurcation, different cutting conditions for extrusion machining AZ31B and 7075-T6 are listed in Table 3 by adjusting the position of constraint. After cutting, chips were collected and embedded into clean resin. The lateral process was mechanically polished, and then the polished sur- faces were etched in the different etching solutions to reveal the deformed microstructures of AZ31B and 7075-T6 (e.g., a $5 \mathrm{~g}$ picric acid $+10 \mathrm{ml}$ water $+10 \mathrm{ml}$ acetic acid $+100 \mathrm{ml}$ ethanol solution lasting for $10 \mathrm{~s}$ for AZ31B and a $2 \% \mathrm{HF}+4 \% \mathrm{HNO}_{3}+94 \% \mathrm{H}_{2} \mathrm{O}$ solution lasting for $5 \mathrm{~s}$ for 7075-T6). These etched specimens were observed with the optical microscope (Olympus BX51M) to examine the morphologies of chips.

\section{Experimental observations}

Figure 3 shows the experimental measurements of cutting forces for different CEFs in extrusion machining AZ31B under the cutting conditions of Table 3. According to Fig. 3, the cutting force fluctuates periodically 


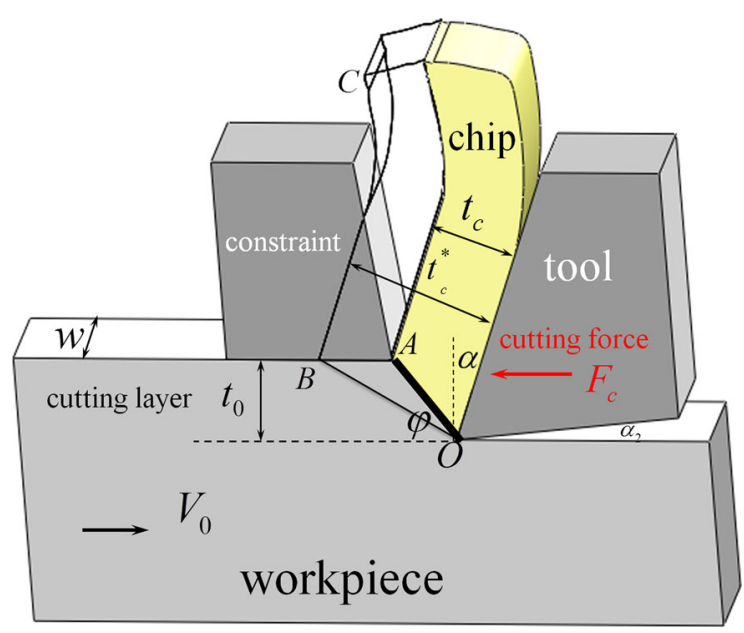

Fig. 2 Schematic of extrusion machining, in which the free machining (FM) is marked by the lines $\mathrm{OBC}$

during FM AZ31B where $\mathrm{CEF}=0$. In spite of the cutting force fluctuation for smaller $\mathrm{CEF}(\mathrm{CEF}=0.28)$, the amplitude of fluctuation and cycle time are smaller than those of FM. The effect of extrusion from constraint increases with the increasing CEF. When CEF reaches or exceeds a critical value, i.e., $\mathrm{CEF}=0.44$, the cutting force cannot fluctuate periodically in extrusion machining AZ31B. The value of cutting force is larger with the increasing CEF during extrusion machining.

In order to reveal the relationship between cutting force fluctuation and the morphology of chips, the chips of AZ31B were etched and observed. The microstructures of chips for different CEFs are shown in Fig. 4. For the case of FM in Fig. 4a, chips tend to segments because of fracture-type deformation within PSZ. As CEF is increased (see in Fig. 4b), chips are still fracture type segments, but the size of segment is smaller than that of FM. As shown in Fig. 4c, d, the morphologies of chips change from fracture-type segments to

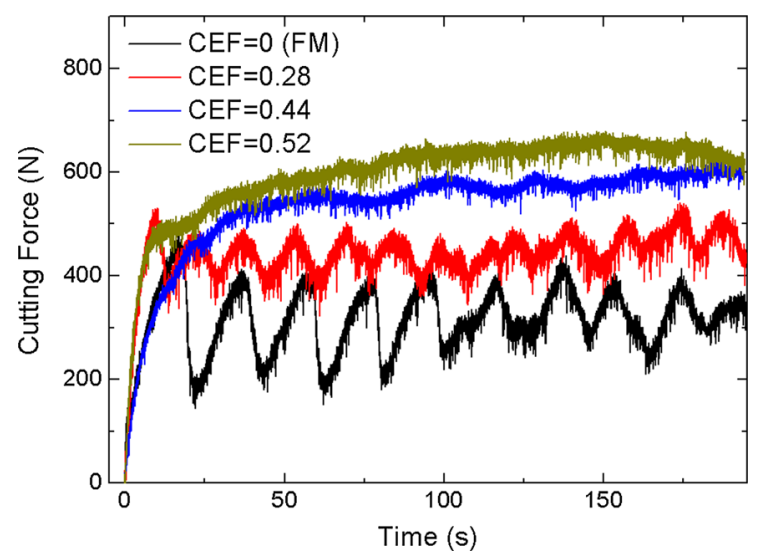

Fig. 3 Experimental measurements of cutting forces for different CEFs in low-speed extrusion machining AZ31B

continuous chip when CEF is further increased. Connecting Figs. 3 with 4, it is found that the deformation mode in extrusion machining determines whether the cutting force fluctuates or not. If the shear deformation in extrusion machining is fracture-type localization (Fig. 4a, b), the cutting force fluctuates periodically as shown in Fig. 3. The CEF in Fig. 4b is larger than CEF in Fig. 4a, so the extrusion level of Fig. 4b is larger than that of Fig. 4a. Compared with Fig. 4a, the suppression of fracture-type localized deformation is more severe in Fig. 4b, which leads to the amplitude of fluctuation and cycle time in Fig. 3 smaller with increasing CEF. When CEF reaches or exceeds a critical value, i.e., $\mathrm{CEF}=0.44$, the shear deformation is homogeneous (Fig. 4c, d). The catastrophic fracture disappears in chips of Fig. 4c, d; therefore, the cutting force cannot fluctuate periodically in Fig. 3.

The experiments for aluminum alloy 7075-T6 listed in Table 3 were conducted to explore universality of the suppression of fracture-type serrated chips during
Table 3 Cutting conditions in extrusion machining magnesium alloy AZ31B and aluminum alloy 7075-T6

\begin{tabular}{llll}
\hline Cutting parameters & Notation & AZ31B & $7075-\mathrm{T6}$ \\
\hline Rake angle & $\alpha$ & $10^{\circ}$ & $10^{\circ}$ \\
Clearance angle & $\alpha_{2}$ & $5^{\circ}$ & $5^{\circ}$ \\
Precut chip thickness & $t_{0}(\mu \mathrm{m})$ & 200 & 200 \\
Cutting width & $w(\mathrm{~mm})$ & 5 & 5 \\
Cutting speed & $V_{0}(\mu \mathrm{m} / \mathrm{s})$ & 10 & 10 \\
Controlled chip thickness & $t_{c}(\mu \mathrm{m})$ & $250,180,140,120$ & $610,550,360,280$ \\
Constraint extrusion factor & $\chi$ & $0,0.28,0.44,0.52$ & $0,0.10,0.41,0.54$
\end{tabular}


Fig. 4 The microstructures of chips for different CEFs in low-speed extrusion machining AZ31B: a $\mathrm{CEF}=0(\mathrm{FM}) ; \mathbf{b}$ $\mathrm{CEF}=0.28 ; \mathbf{c} \mathrm{CEF}=0.44$; d $\mathrm{CEF}=0.52$
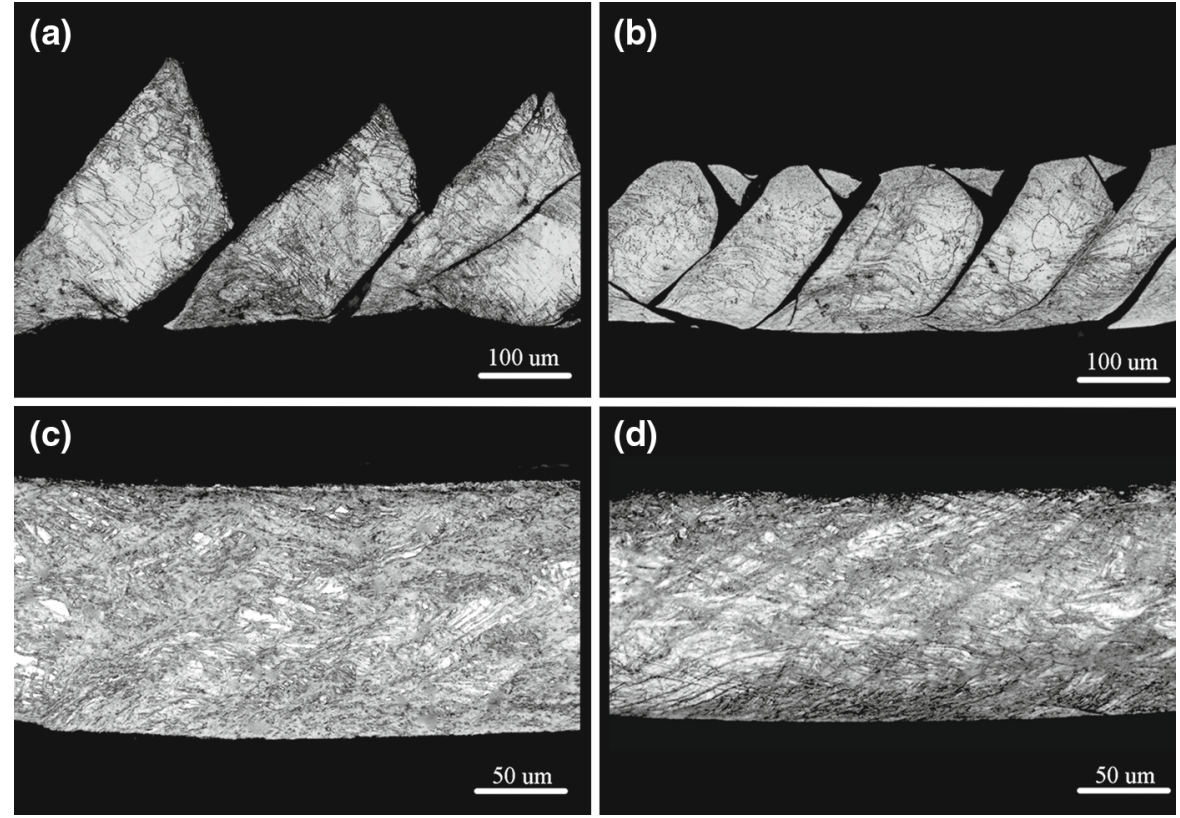

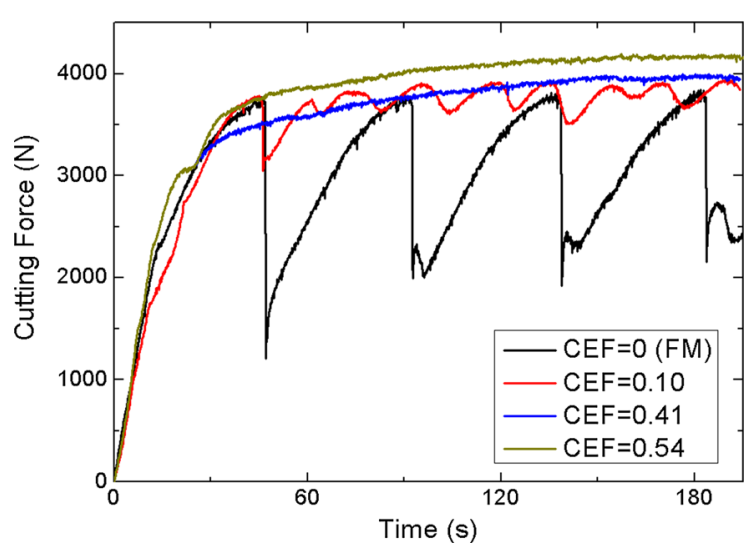

Fig. 5 Experimental measurements of cutting forces for different CEFs in low-speed extrusion machining 7075-T6

machining other materials. The measurements of cutting forces and the microstructures of chips for different CEFs during extrusion machining 7075-T6 are illustrated in Figs. 5 and 6, respectively. As seen in Figs. 5 and 6, the cutting forces fluctuate periodically and the chips are fracture-type segments if the CEF is smaller than a certain value; however, the cutting forces do not fluctuate periodically and the chips are continuous sheet when the CEF reaches or exceeds a critical value. Comparing the experimental results in extrusion machining AZ31B with those in extrusion machining 7075-T6, we find that the tendencies of cut- ting forces and chip morphologies with increasing CEF are the same, but the critical CEF distinguishing fracture type serrated chip from continuous chip depends on the machining materials.

\section{Fracture-based model for extrusion machining}

\subsection{The governing equations}

As seen in Figs. 4 and 6, the fracture leads to the segmented chips during extrusion machining AZ31B and 7075-T6 at the cutting speed of $10 \mu \mathrm{m} / \mathrm{s}$. The existing theoretical model is based on shear band type deformation which is the typical phenomenon in high-speed machining $[10,11,31]$. The shear band-based models cannot explain the chip formation in extrusion machining AZ31B and 7075-T6 at a relatively low cutting speed. Here, based on the experimental observation of extrusion machining AZ31B and 7075-T6, a fracturebased model for extrusion machining is proposed to characterize the process of extrusion machining.

Figure 7 shows the local stress state around PSZ. Because of the relative motion of the tool-workpiece system, we assume that the workpiece is static and the tool is moving toward the workpiece at a speed of $V_{0}$. The deformation of the workpiece ahead of the tool concentrates within PSZ. The width $h$ of PSZ is assumed to $t_{0} / 10[1,33]$. The tool and constraint exert 
Fig. 6 The microstructures of chips for different CEFs in low-speed extrusion machining 7075-T6: a $\mathrm{CEF}=0(\mathrm{FM}) ; \mathbf{b}$ $\mathrm{CEF}=0.10 ; \mathbf{c} \mathrm{CEF}=0.41$; d $\mathrm{CEF}=0.54$
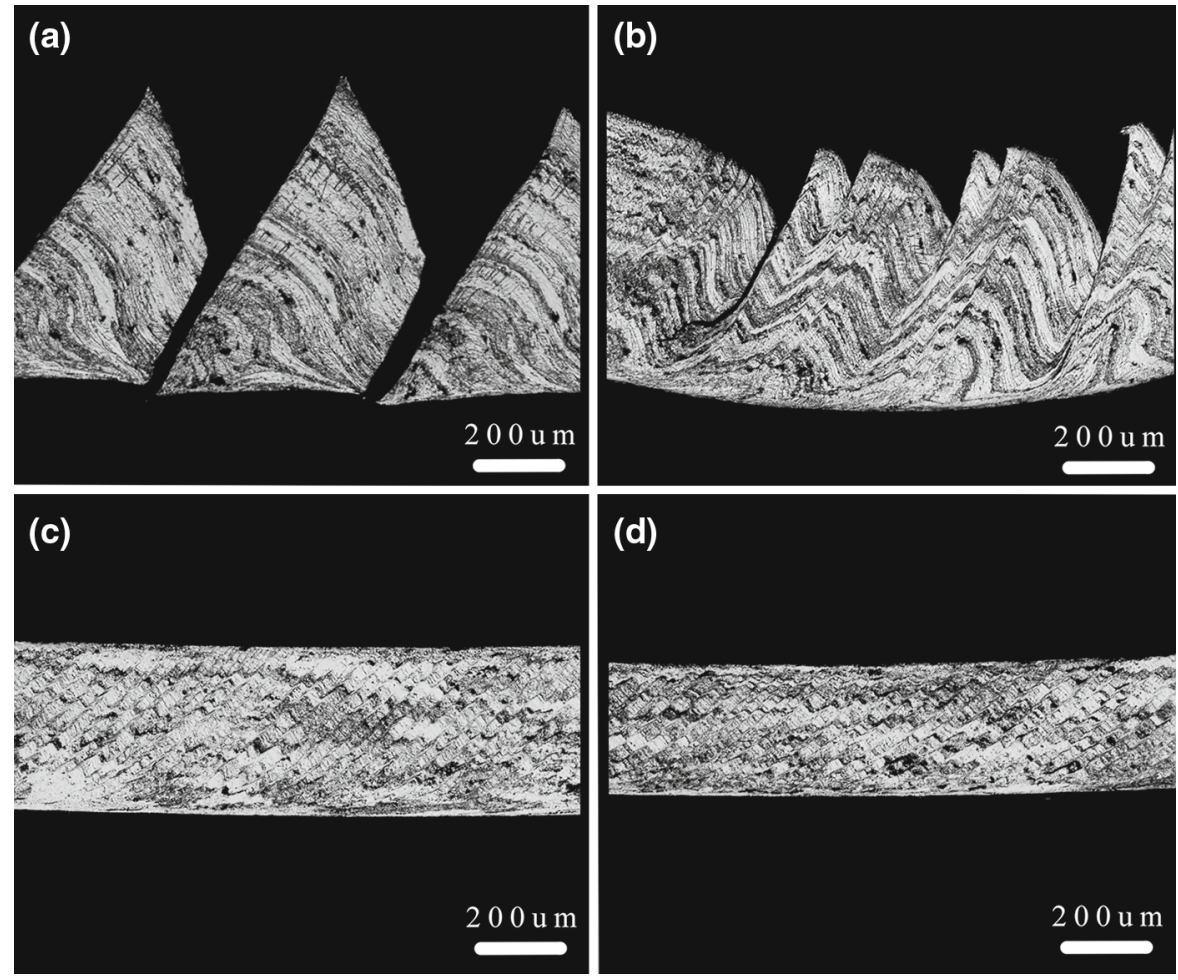

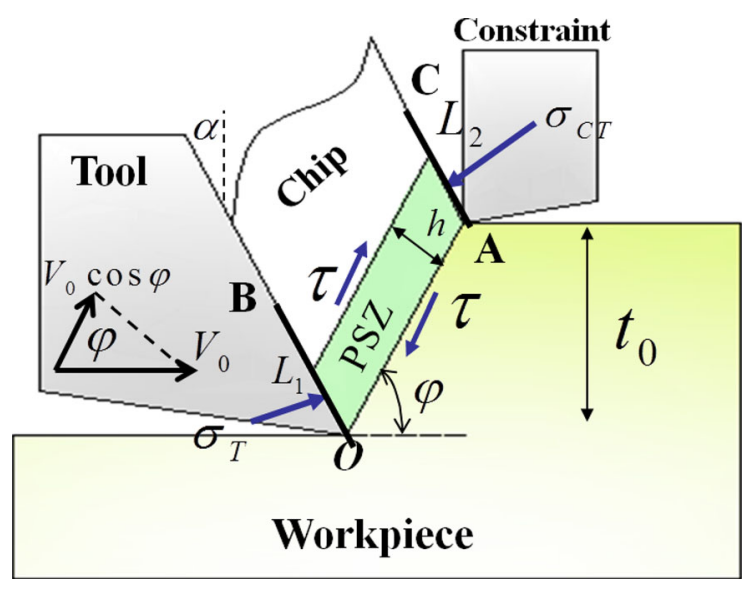

Fig. 7 Schematic of the extrusion machining model in which the tool is moving toward the workpiece relatively

forces on the workpiece, which cause the precut layer of depth $t_{0}$ to move out of the rake face in the form of a chip with a thickness $t_{c}$. Based on the work of Burns and Davies [10], the deformation process in PSZ is treated as a local compression. Thus, the evolution equation for tool compressive stress $\sigma_{T}$ and constraint compression stress $\sigma_{C T}$ is given by:
$\frac{\mathrm{d}\left(\sigma_{T}-\sigma_{C T}\right)}{\mathrm{d} t}=\frac{E \sin \varphi}{t_{0}}\left(V_{0} \cos \varphi-V\right)$

where $E$ is the elastic modulus of the workpiece material, $V_{0} \cos \varphi$ is the velocity component of the tool along PSZ and $V$ is the speed of plastic flow in PSZ. The local compressive stress $\sigma_{T}$ and $\sigma_{C T}$ lead to a shear stress $\tau$ to build up in PSZ. Ignoring small inertial terms and because of the momentum balance, it is required that

$\sigma_{T} L_{1} w=\sigma_{C T} L_{2} w+\frac{\tau w t_{0}}{\sin \varphi}$,

where $L_{1}$ and $L_{2}$ are the contact length of the local compressive stress $\sigma_{T}$ and $\sigma_{C T}$, respectively, and $w$ is the width of workpiece. In order to highlight the essential physical argument, we simplify the geometry by assuming that $L_{1}=L_{2}$. Combining Eq. (1) with Eq. (2), the following form is obtained:

$\frac{\mathrm{d} \tau}{\mathrm{d} t}=\frac{E L_{1} \sin ^{2} \varphi}{t_{0}^{2}}\left(V_{0} \cos \varphi-V\right)$.

The shear stress causes the materials in PSZ to deform elastically at the initial stage of extrusion machining; therefore, the speed of plastic flow $V=0$. 
If the shear stress $\tau$ exceeds the yield stress $\tau_{y}$ of materials, plastic flow will happen and there will be a plastic shear strain rate $\dot{\gamma}$ in PSZ:

$\dot{\gamma}=\frac{V}{h}$.

Based on the experimental results in Fig. 4, the plastic work is partly converted into fracture energy, so the heat balance equation is shown as the following form:

$\rho c \frac{\mathrm{d} T}{\mathrm{~d} t}=\beta \tau \dot{\gamma}+\rho c V_{0} \sin \varphi \frac{T_{a}-T}{h}+4 k \frac{T_{a}-T}{h^{2}}$,

where $\beta$ is Taylor-Quinney coefficient, $T$ is the temperature in PSZ, $T_{a}$ is ambient temperature, and $\rho, c, k$ are density, specific heat capacity, thermal conductivity of the workpiece material, respectively. It is found from Eq. (5) that there are three different physical processes altering the temperature in PSZ: heat generation due to plastic working (the first term in the right side of the equation), convection and diffusion of heat (the second and third terms, respectively).

If all of the plastic work is dissipated as heat, TaylorQuinney coefficient $\beta=1$ [10,34-36]. However, the plastic work is not entirely converted into heat in fact [37-39]. The $\beta$ percent of plastic work is dissipated as heat and the rest of plastic work is dissipated in other way. Here, based on the observation of microstructures in Fig. 4, it is assumed that $(1-\beta)$ percent of plastic work is dissipated as fracture. From energy consideration, Dolinski and Rittel [40], Dolinski et al. [41] introduced a damage parameter $D(D \in[0,1])$ to model the damage evolution. For $D=0$, there is no damage in materials; and the materials completely damage if $D=1$. In order to examine the effect of fracture on the chip formation in extrusion machining, not only the Hillerborg's fracture energy $G_{f}[42]$ but also the convection and diffusion are assumed to have an influence on the damage parameter $D$. The balance about $D$ in PSZ is given by:

$$
\begin{aligned}
\frac{\mathrm{d} D}{\mathrm{~d} t}= & (1-\beta)(1-D) \frac{\sqrt{3} \tau V}{G_{f}}-V_{0} \sin \varphi \frac{D}{h} \\
& -4 \tilde{\chi}_{D} \frac{D}{h^{2}} .
\end{aligned}
$$

Here, $\tilde{\chi}_{D}$ is the diffusion coefficient of damage evolution and diffusion is approximated by a second-order difference over the primary shear zone. $\tilde{\chi}_{D}$ is equal to $\tilde{l}^{2}\left(V_{0} \sin \varphi\right)^{2} / \tilde{\eta}$ where $\tilde{l}$ is the damage diffusion length scale on the order of a grain radius and $\tilde{\eta}$ is the diffusion coefficient of the crystal defect. Hillerborg's fracture energy $G_{f}$ is determined by $G_{f}=K_{C}^{2}\left(1-v^{2}\right) / E$ where $K_{C}$ is the fracture toughness and $v$ is Poisson's ratio [43]. Equation(6) states that the rate of damage variation in PSZ is governed by partial heat production of plastic working (the first term in the right side of the equation), convection and diffusion of damage (the second and third terms, respectively).

The degradation of material is inevitable if there is damage of fracture in material. Thus, based on the thermo-viscoplastic material model, a new material constitutive equation containing the effect of fracture is required:

$\tau=f(\gamma, \dot{\gamma}, T, D)$,

where $\gamma, \dot{\gamma}, T$ and $D$ are shear strain, shear strain rate, temperature and damage parameter in PSZ, respectively.

Based on the derivation above, the governing system of extrusion machining is determined by the following ordinary differential equations (ODE):

$$
\left\{\begin{array}{l}
\frac{\mathrm{d} \tau}{\mathrm{d} t}=\frac{E L_{1} \sin ^{2} \varphi}{t_{0}^{2}}\left(V_{0} \cos \varphi-V\right) \\
\tan \varphi=\frac{t_{0} \cos \alpha}{t_{c}^{*}(1-\chi)-t_{0} \sin \alpha} \\
\dot{\gamma}=\frac{\mathrm{d} \gamma}{\mathrm{d} t}=\frac{V}{h} \\
\rho c \frac{\mathrm{d} T}{\mathrm{~d} t}=\beta \tau \dot{\gamma}+\rho c V_{0} \sin \varphi \frac{T_{a}-T}{h}+4 k \frac{T_{a}-T}{h^{2}} \\
\frac{\mathrm{d} D}{\mathrm{~d} t}=(1-\beta)(1-D) \frac{\sqrt{3} \tau V}{G_{f}}-\frac{V_{0} \sin \varphi}{h} D-\frac{4 \hat{l}^{2}\left(V_{0} \sin \varphi\right)^{2}}{\tilde{\eta} h^{2}} D \\
\tau=f(\gamma, \dot{\gamma}, T, D)
\end{array} .\right.
$$

In Eq. (8), the constant parameters are $E, L_{1}, \varphi, V_{0}$, $t_{0}, \alpha, t_{c}^{*}, \chi, h, \rho, c, \beta, T_{a}, k, G_{f}, \tilde{l}$ and $\tilde{\eta}$. The dynamic variables of Eq. (8) are $\tau, V, \dot{\gamma}, T, D$ and $\gamma$. Specifically, the cutting speed $V_{0}$ and CEF $\chi$ are chosen as bifurcation parameters to analyze the occurrence of Hopf bifurcation during extrusion machining.

The initial condition (IC) governing the one-dimensional extrusion machining is given as the following form:

$I C:\left\{\begin{array}{l}\tau(0)=0 \\ T(0)=T_{a} . \\ D(0)=0\end{array}\right.$.

If the specific form of constitutive relation Eq. (7) is given, we can solve simultaneously the coupled govern- 

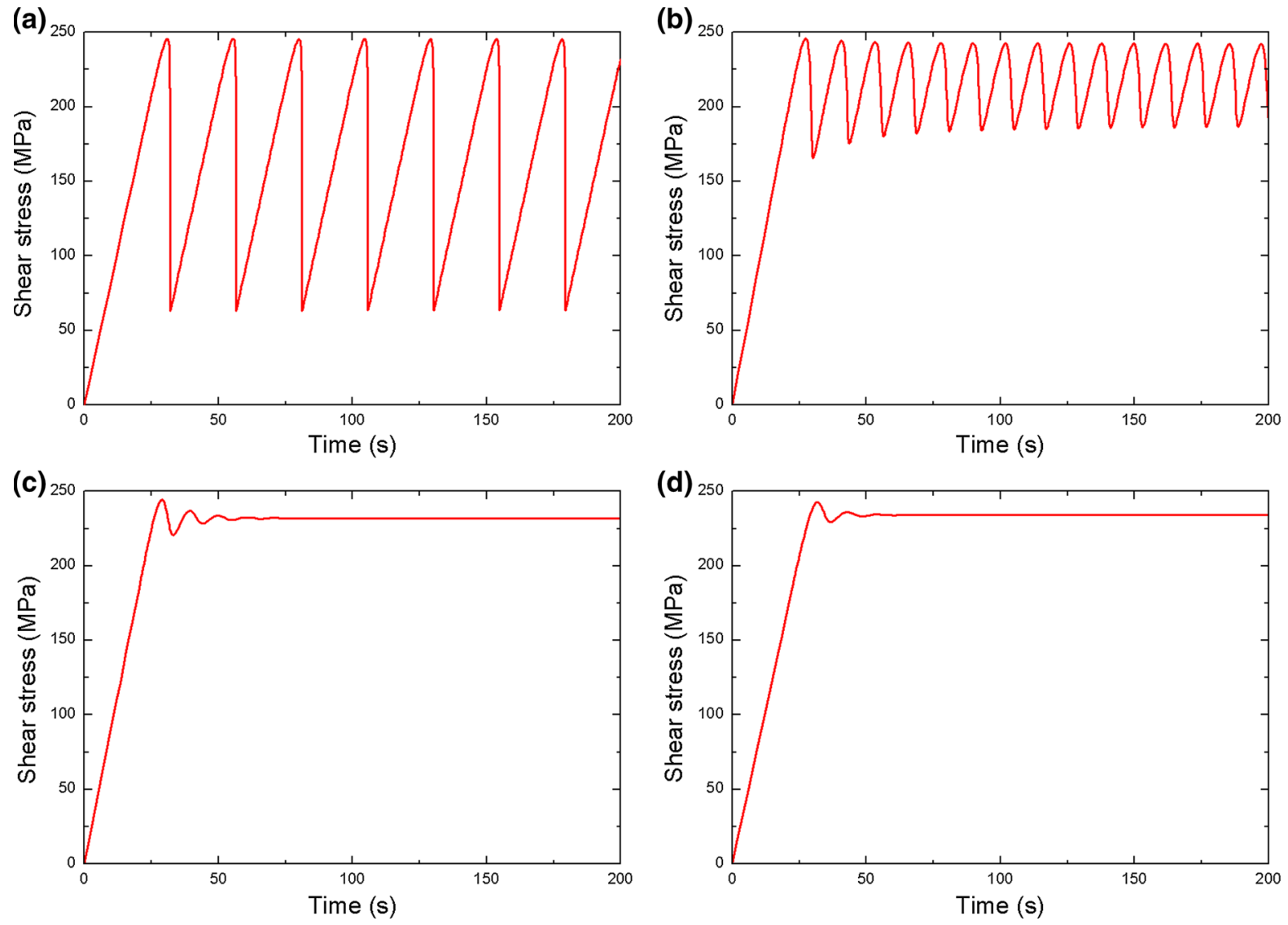

Fig. 8 Considering the fracture-type damage, the relationship between the shear stress and time during low-speed extrusion machining AZ31B for different CEFs: $\mathbf{a} \mathrm{CEF}=0(\mathrm{FM}) ; \mathbf{b} \mathrm{CEF}=0.28 ; \mathbf{c} \mathrm{CEF}=0.44 ; \mathbf{d} \mathrm{CEF}=0.52$

ing Eqs. (8)-(9) of shear stress, shear strain, shear strain rate, temperature and damage parameter in course of extrusion machining.

\section{Comparison of model and experimental measurements}

The workpiece material is assumed to be thermoviscoplastic. Considering the effect of damage evolution on material behavior, the modified Johnson-Cook (J-C) law is chosen to be the constitutive law of the workpiece material:

$$
\begin{aligned}
\tau= & \frac{1}{\sqrt{3}} \tau_{y}\left[1+C \ln \left(\frac{\dot{\bar{\gamma}}}{\dot{\bar{\gamma}}}\right)\right] \\
& {\left[1-\left(\frac{T-T_{a}}{T_{m}-T_{a}}\right)^{m}\right](1-D) . }
\end{aligned}
$$

Here, the strain hardening is ignored and $\tau_{y}$ is the shear yield strength of material. The parameters such as $C, \dot{\gamma}_{0}, m, T_{a}$ are listed in Table 2. $\left[1+C \ln \left(\frac{\dot{\gamma}}{\dot{\bar{\gamma}}}\right)\right]$, $\left[1-\left(\frac{T-T_{a}}{T_{m}-T_{a}}\right)^{m}\right]$ and $(1-D)$ represent the effect of strain rate hardening, thermal softening and damage softening, respectively. Substituting Eq.(10) into Eqs. (8)-(9), the time evolutions of the shear stress $\tau$, temperature $T$ and damage parameter $D$ are obtained by solving the system of ordinary differential Eqs. (8)(9). Figures 8, 9 and 10 show the relationship between shear stress, temperature, damage parameter and time during extrusion machining AZ31B for different CEFs.

As seen in Figs. 8, 9 and 10, the periodic oscillations of shear stress, temperature and damage parameter disappear gradually with the increasing CEF. The amplitude of fluctuation and cycle time decrease with the increasing $\mathrm{CEF}$, and there is no periodic oscilla- 

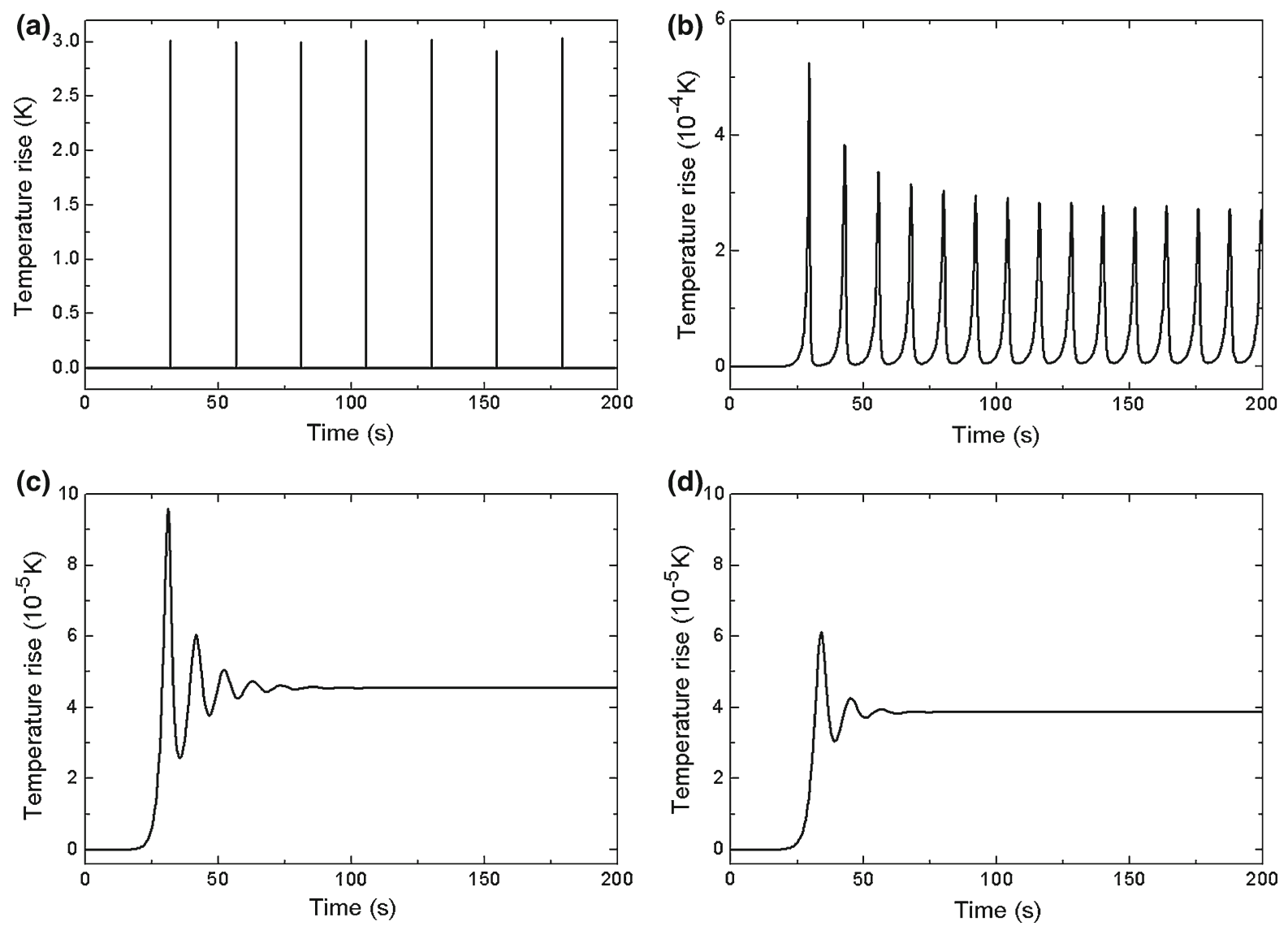

Fig. 9 Considering the fracture-type damage, the relationship between the temperature rise and time during low-speed extrusion machining AZ31B for different CEFs: $\mathbf{a} \mathrm{CEF}=0(\mathrm{FM}) ; \mathbf{b} \mathrm{CEF}=0.28 ; \mathbf{c} \mathrm{CEF}=0.44 ; \mathbf{d} \mathrm{CEF}=0.52$

tion if CEF exceeds a certain value. The temperature rise in low-speed extrusion machining is $<3 \mathrm{~K}$, so the effect of thermal softening is of no importance. The damage parameter is smaller for larger CEF, which means that the constraint restrains the fracture-type damage during extrusion machining process with large CEF.

By contrast, the workpiece material is only assumed to be thermo-viscoplastic in the model where the effect of damage evolution on material behavior is not taken into consideration. The constitutive law of the workpiece material is the following form:

$\tau=\frac{1}{\sqrt{3}} \tau_{y}\left[1+C \ln \left(\frac{\dot{\gamma}}{\dot{\bar{\gamma}}_{0}}\right)\right]\left[1-\left(\frac{T-T_{a}}{T_{m}-T_{a}}\right)^{m}\right]$.

Equation(6) is also ignored because the damage evolution is not considered in the model. Substituting Eq.(11) into Eqs.(3)-(5) and (9), the relation- ships between shear stress, temperature and time for different CEFs are illustrated in Figs. 11, 12. It is found that the temperature rise is $<1 \mathrm{~K}$ and the thermal softening do not lead to the instability of low-speed extrusion machining. The shear stress and temperature do not fluctuate periodically for all CEFs during low-speed extrusion machining AZ31B if the effect of fracture-type damage is not taken into consideration.

In order to distinguish whether the fracture-type damage is taken into consideration or not, the results of two theoretical models are compared with the measurements of extrusion machining experiments. Based on Merchant circle [44,45], the relationship between cutting force $F_{c}$ and shear stress $\tau$ is obtained where the thrust force is ignored for simplicity:

$\frac{\tau t_{0} w}{\sin \varphi}=F_{c} \cos \varphi$ 

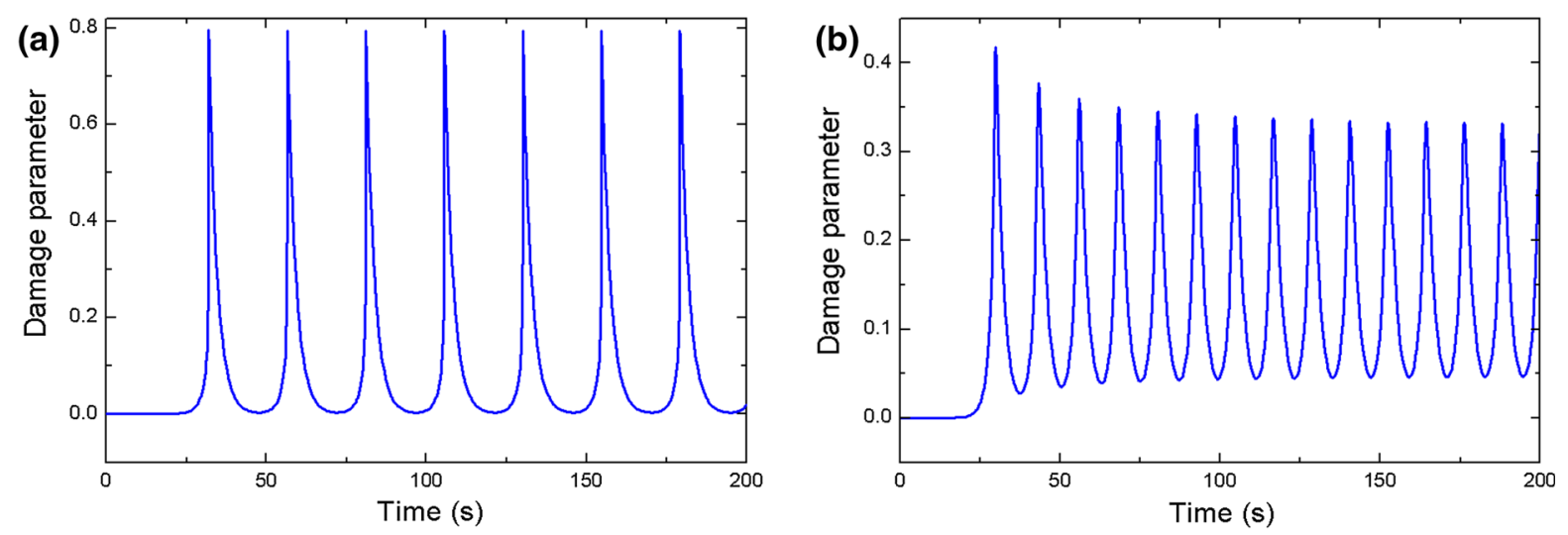

(c)
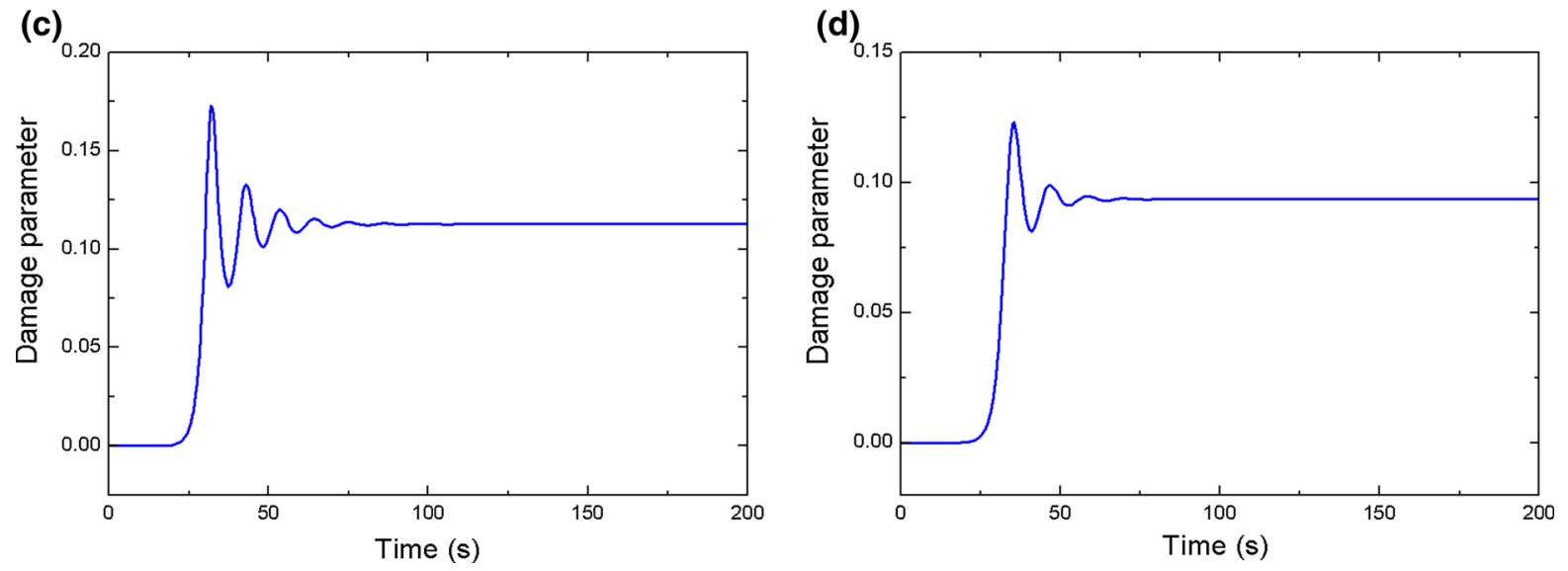

Fig. 10 Considering the fracture-type damage, the relationship between the damage parameter and time during low-speed extrusion machining AZ31B for different CEFs: $\mathbf{a} \mathrm{CEF}=0(\mathrm{FM}) ; \mathbf{b} \mathrm{CEF}=0.28 ; \mathbf{c} \mathrm{CEF}=0.44 ; \mathbf{d} \mathrm{CEF}=0.52$

Then, the predicted cutting forces are obtained by substituting the calculative shear stress $\tau$ into Eq. (12).

Figure 13 shows the comparison of cutting forces between different models and experimental measurements for different CEFs during extrusion machining AZ31B. In terms of cutting force, the theoretical model of damage is identical with the cutting force of experimental measurements; however, the theoretical model without damage cannot reveal the periodic oscillations of cutting force. The cutting forces predicted by the model of damage fluctuate periodically for small CEF (see Fig. 13a, b), which is the same as the experimental measurements. If the CEF exceeds a certain value (see in Fig. 13c, d), there is no periodic oscillation of cutting force in both the model of damage and experimental results. Compared with cutting forces predicted by the model of damage, the cutting forces of the model without damage are much larger than the experimental measurements for all the CEFs (see in Fig. 13); more- over, the model without damage cannot explain the cutting force fluctuation of experiments in essence (see in Fig. 13a, b). According to the analysis above, the model without damage predicts the upper limit of the cutting force. Compared with the model without damage, the theoretical model of damage is more reasonable to explain the phenomenon in extrusion machining experiments.

In order to confirm that the model with fracture-type damage is universal, the cutting forces predicted by the present model are compared with the experimental measurements during extrusion machining 7075-T6. The shear stress $\tau$ in PSZ during extrusion machining 7075-T6 is calculated by substituting the mechanical parameters in Table 2 and machining parameters in Table 3 into Eqs. (3)-(11). Then, the predicted cutting forces during extrusion machining 7075-T6 are shown in Fig. 14 by substituting the calculative shear stress $\tau$ into Eq. (12). Compared with the model without dam- 

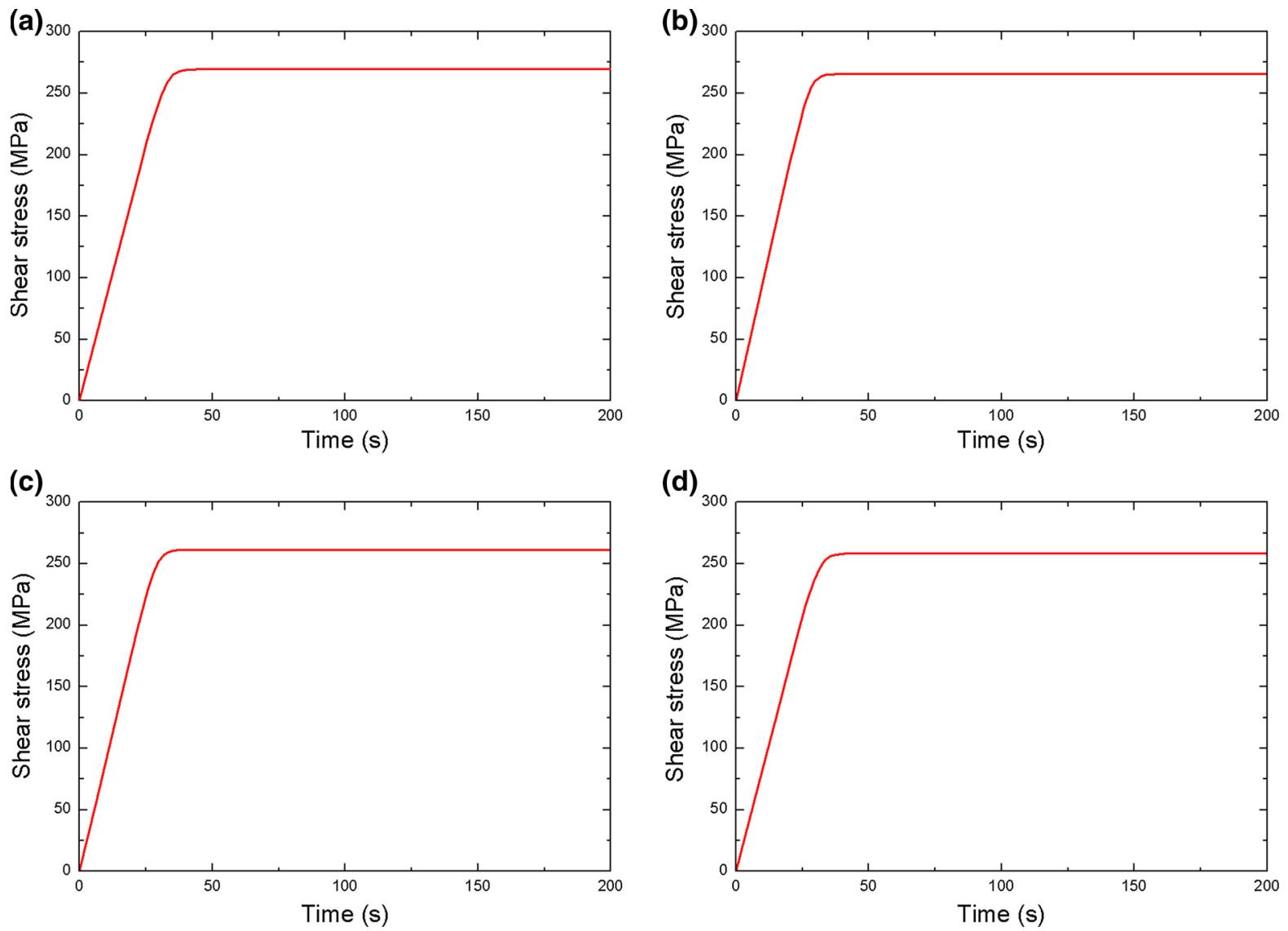

Fig. 11 Ignoring the fracture-type damage, the relationship between the shear stress and time during low-speed extrusion machining AZ31B for different CEFs: $\mathbf{a} \mathrm{CEF}=0(\mathrm{FM}) ; \mathbf{b} \mathrm{CEF}=0.28 ; \mathbf{c} \mathrm{CEF}=0.44 ; \mathbf{d} \mathrm{CEF}=0.52$

age, the theoretical model of damage is more reasonable to predict the cutting force in extrusion machining 7075-T6. Therefore, the present model of damage is independent on the machining materials and can be widely used to elucidate the mechanism of deformation in extrusion machining.

\section{Discussion of the fracture-based model}

\subsection{Effect of cutting speed on Hopf bifurcation in FM}

Equations (8)-(9) constitute the governing equations of extrusion machining. For free machining (FM), there is no extrusion effect of constraint to change the shear angle $\varphi$. The effects of thermal softening and fracturetype damage on the process of machining are analyzed by varying the cutting speed $V_{0}$ in free machining. Sub- stituting the material parameters and machining parameters into the governing equations of FM model, the variations of shear stress $\tau$, temperature $T$ and damage parameter $D$ in FM at a wide range of cutting speeds are shown in Fig. 15. For high-speed machining in Fig. 15a1, the shear stress fluctuates periodically which is the same as the trend of cutting forces in the available experimental measurements [46-48]. Moreover, more and more researchers have confirmed that the periodical oscillation is the result of shear band type localized deformation in high-speed machining $[26,27,49]$. The local temperature rise is about $400 \mathrm{~K}$, but the increment of damage parameter is $<0.015$ in high-speed FM (Fig. 15a2, a3). Compared with fracture type deformation, shear band type deformation is more dominating in high-speed machining. The calculated temperature and damage parameter from the present theoretical model demonstrate that shear band 

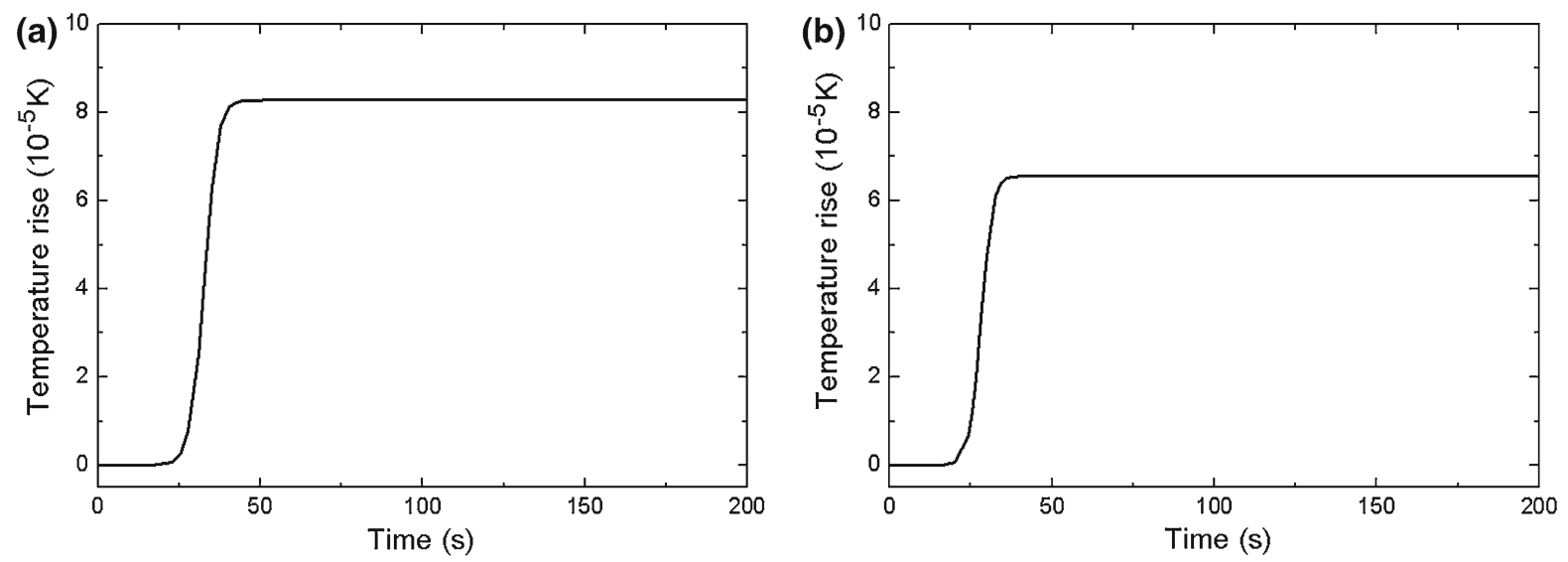

(c)
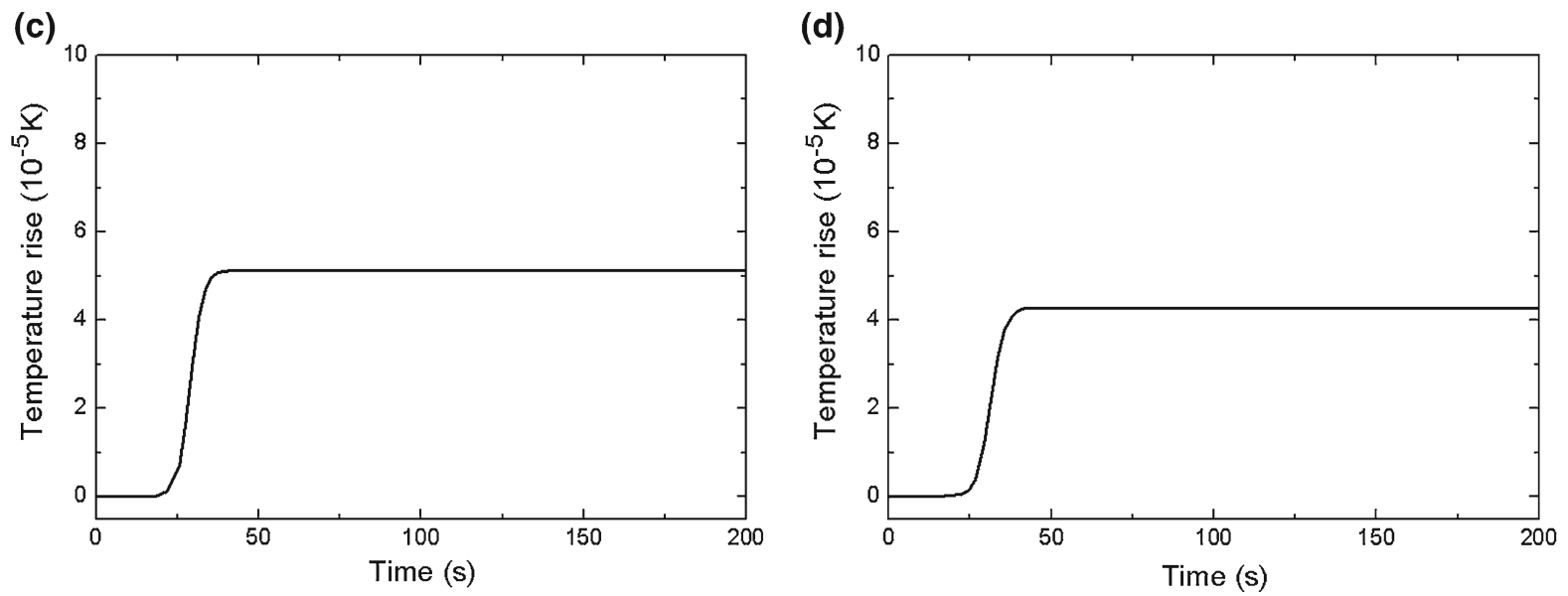

Fig. 12 Ignoring the fracture-type damage, the relationship between the temperature rise and time during low-speed extrusion machining AZ31B for different CEFs: $\mathbf{a} \mathrm{CEF}=0(\mathrm{FM}) ; \mathbf{b} \mathrm{CEF}=0.28 ; \mathbf{c} \mathrm{CEF}=0.44 ; \mathbf{d} \mathrm{CEF}=0.52$

type deformation leads to the formation of serrated chips in high-speed FM. For low-speed machining in Fig. 15b, c, the shear stress does not fluctuate periodically, the maximal temperature rise is $<2 \mathrm{~K}$, and the maximal increment of damage parameter is $<0.03$. Neither shear band type deformation nor fracture-type deformation happens in the chip formation during lowspeed machining where the cutting speed $V_{0}$ is close to the range of the cutting speeds in Fig. 15b, c. The deformation in chips predicted by the presented model is homogeneous in low-speed FM, which is identical with the available experimental observations [29,5052]. If the cutting speed is further slowed down (e.g., $V_{0}=10^{-5} \mathrm{~m} / \mathrm{s}$ ), the calculated results in Fig. $15 \mathrm{~d} 1$ show that the cutting force should fluctuate periodically in FM, which is certified by the experimental measurements in Fig. 3. As illustrated in Fig. 15d2, d3, the maximal increment of damage parameter is about 0.8 , but the maximal temperature rise is $<3 \mathrm{~K}$. The presented theoretical model reveals that fracture-type deformation is the primary deformation mode during relatively low-speed FM, which is in accordance with low-speed machining experiments in Fig. 4.

Ignoring the fracture-type damage in FM, Burns and Davies [10] first put forward the thermal-softeningbased model to reveal the mechanism of chip segmentation in high-speed FM. The model of Burns and Davies is successful to explain the transition from shear band type localized deformation to homogeneous deformation with the decreasing cutting speed in FM. However, if the cutting speed is below a certain value (e.g., $10^{-5} \mathrm{~m} / \mathrm{s}$ ), the calculated results of the thermalsoftening-based model show the shear stress $\tau$ do not fluctuate periodically in FM (Fig. 11). Therefore, the thermal-softening-based model of Burns and Davies is invalid to explain the periodic oscillation of cut- 

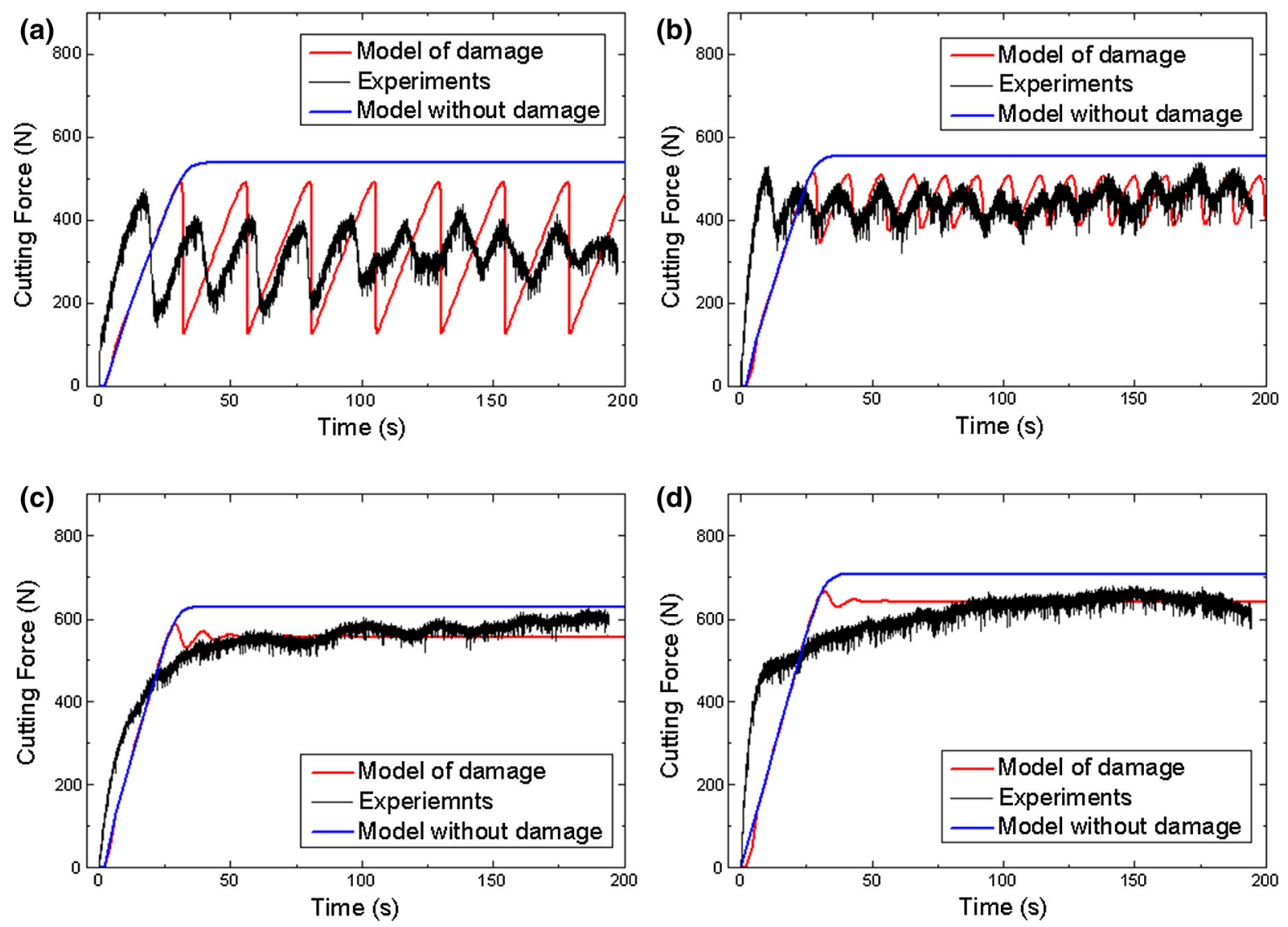

Fig. 13 Comparison of cutting forces between the different models and experimental measurements for different CEFs during extrusion machining $\mathrm{AZ31B}$ : $\mathbf{a} \mathrm{CEF}=0(\mathrm{FM}) ; \mathbf{b} \mathrm{CEF}=0.28 ; \mathbf{c} \mathrm{CEF}=0.44 ; \mathbf{d} \mathrm{CEF}=0.52$

ting forces and segmented chips in low-speed FM. Not only the thermal-softening deformation but also fracture-type deformation is taken into consideration in the present model. When the cutting speed changes from high speed to low speed, the presented model is valid to reveal the transition from shear band type localized deformation to homogeneous deformation (see in Fig. 15a-c). Especially for low-speed FM, compared with the thermal-softening-based model of Burns and Davies in Fig. 11a, the fracture-based model in Fig. 15d is more successful to explain the experimental phenomenon in Figs. 3 and 4. For a relatively high cutting speed as shown in Fig. 15a3-c3, the increment of damage parameter is $<0.03$, so the present model shows fracture-type deformation is negligible. Therefore, it is reasonable for Burns and Davies to ignore the effect of fracture-type deformation for a relatively high cutting speed. The present theoretical model not only degrades into the model of Burns and Davies for relatively high-speed FM, but also explains the experimental phenomenon of low-speed FM.

\subsection{Effect of CEF on Hopf bifurcation}

According to the discussion above, fracture-type deformation is less important than the thermal-softeningtype deformation for high-speed extrusion machining. Therefore, the thermal-softening-based model is sufficient to characterize the formation of chips in highspeed extrusion machining. Detail discussions on the transition from shear band type localized deformation to homogeneous deformation in high-speed extrusion machining have been made in the literature [31]. As for low-speed extrusion machining, the thermalsoftening-based model without fracture-type damage is useless to characterize the periodic oscillation of cutting forces (see in Fig. 13a, b). There is another defor- 

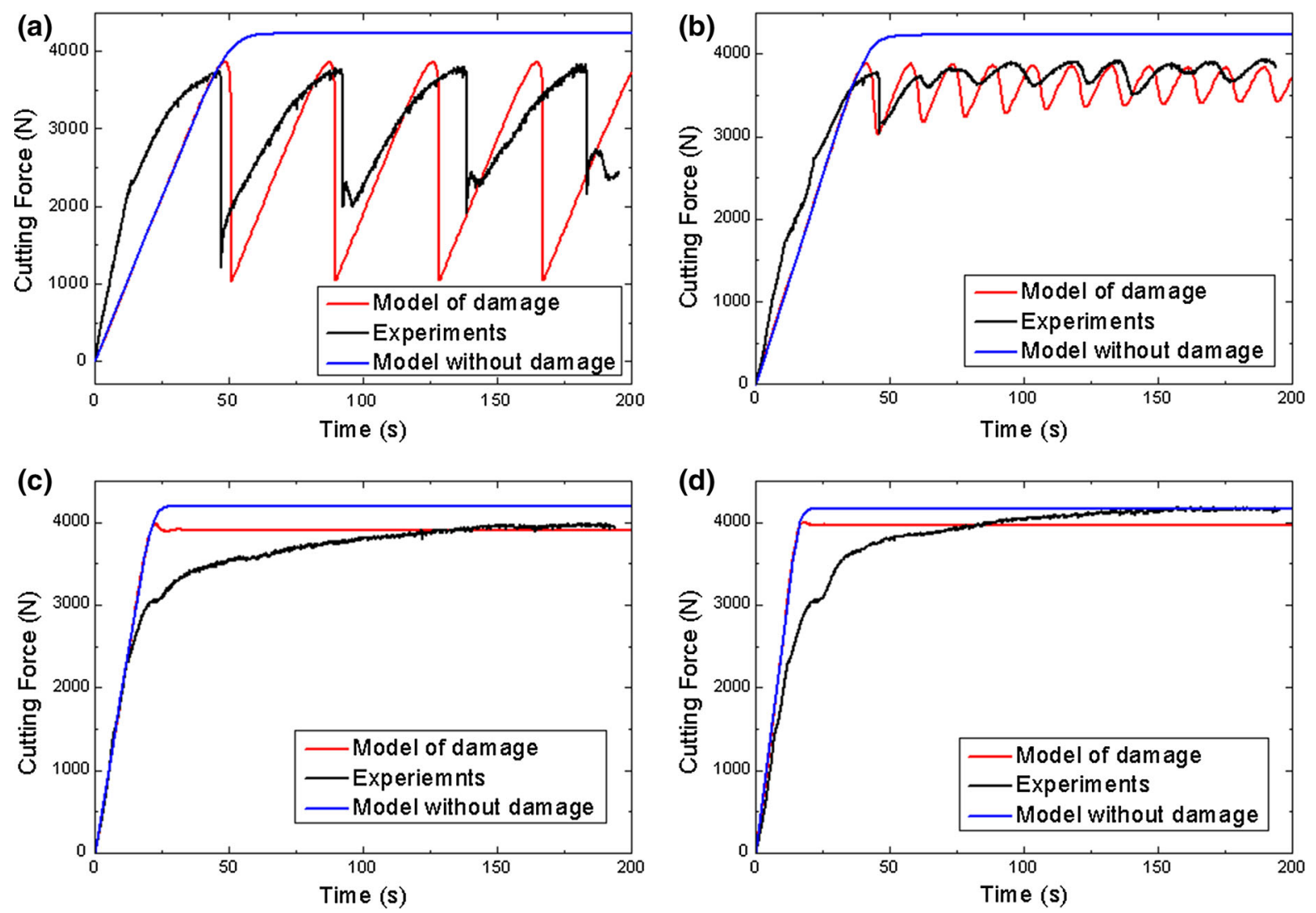

Fig. 14 Comparison of cutting forces between the different models and experimental measurements for different CEFs during extrusion machining 7075-T6: a $\mathrm{CEF}=0(\mathrm{FM}) ; \mathbf{b} \mathrm{CEF}=0.10 ; \mathbf{c} \mathrm{CEF}=0.41 ; \mathbf{d} \mathrm{CEF}=0.54$

mation mechanism controlling the formation of chips in low-speed extrusion machining. Based on the experimental observations in Fig. 4, a fracture-based model which combines the thermal-softening-type deformation with fracture type deformation is proposed here to explain experimental phenomenon in Fig. 4. The trajectories of shear stress $\tau$, temperature $T$ and damage parameter $D$ in low-speed extrusion machining are illustrated in Fig. 16 by using the calculated results in Figs. 8, 9 and 10. The theoretical calculations, together with the experimental observations, provide us with an insight into the physical picture of chip formation during low-speed extrusion machining. During extrusion machining process, shear stress in materials entering PSZ initially increases quickly. The source of fracture damage exceeds its convection and diffusion with continuous increase in shear stress, which finally leads to drastic creation of fracture in chips. As shown in the experimental observations of Fig. 4, for small CEF in
Fig. 4a, b, the initiation and propagation of fracture will decrease the shear stress in PSZ due to energy dissipation (see in Fig. 8a, b). The decreased shear stress slows down the fracture source, whereas the material moving out of PSZ and new material moving into PSZ speeds up the fracture disappearance in PSZ (Fig. 10a, b). At the same time, the heat production also ceases and the heat dissipation cools the PSZ. Unlike high-speed extrusion machining, the maximal temperature rise is $<3 \mathrm{~K}$ (Fig. 9a, b), so the equilibrium of the heat production and dissipation is less important in the chip formation of low-speed extrusion machining. Soon, enough new material without fracture damage passes through the PSZ to increase the shear stress again. As a consequence, the fracture type damage and temperature build up again; therefore, the machining cycle repeats itself all the time. As we have shown previously in Figs. 8a, b, 9 and 10a, b, this provides automatic feedback in shear stress $\tau$, temperature $T$ and damage parameter 
(a1)
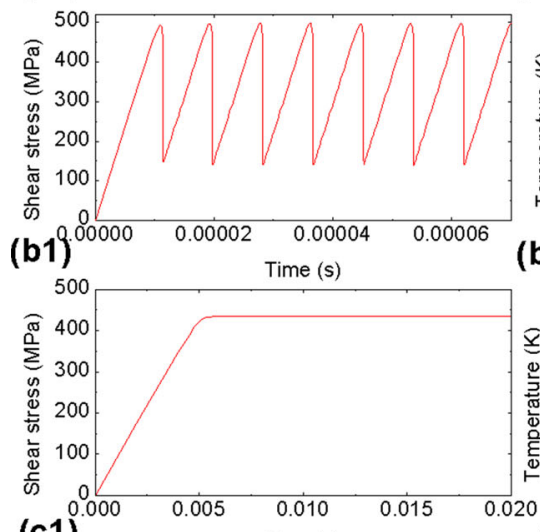

(c1)
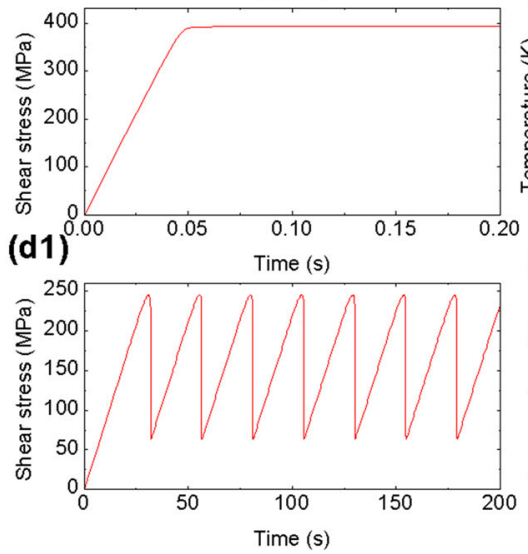

(a2)

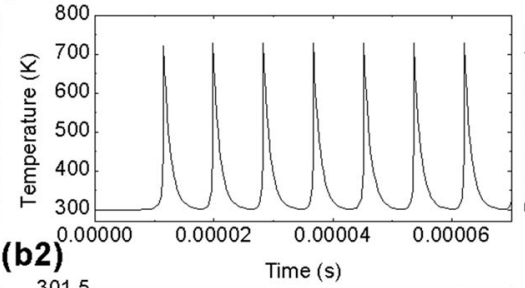

(b2)

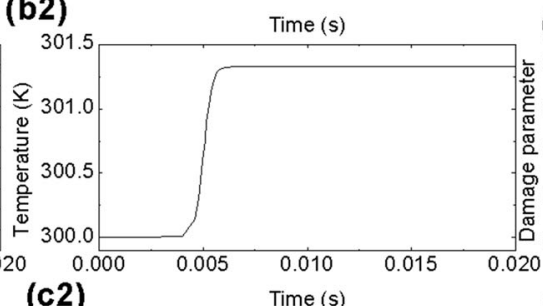

(c2)

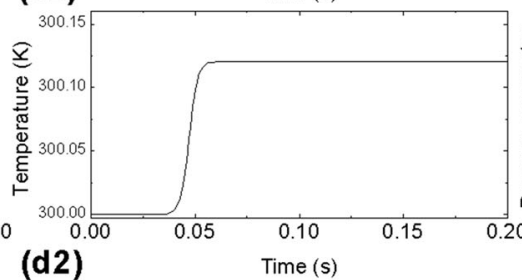

(d2)

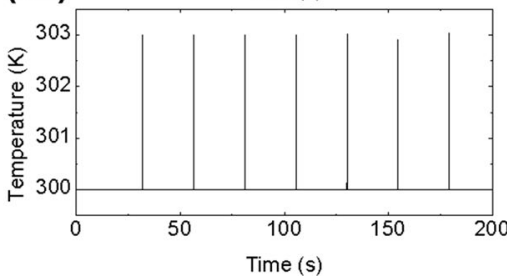

(a3)

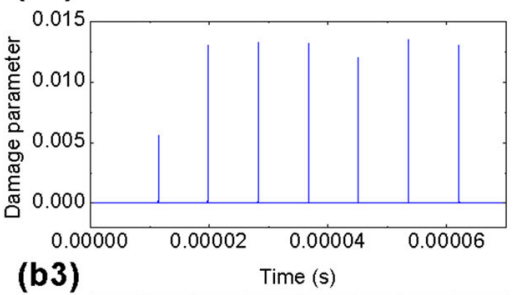

(b3)

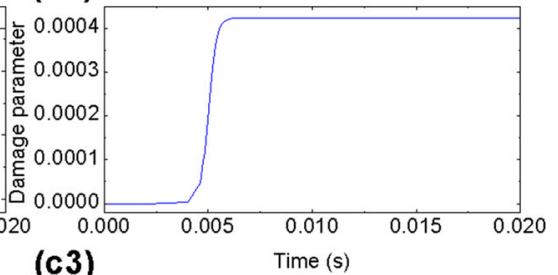

(c3)

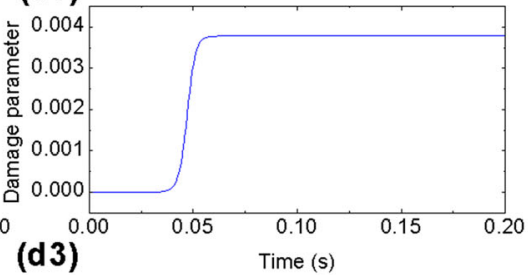

(d3)

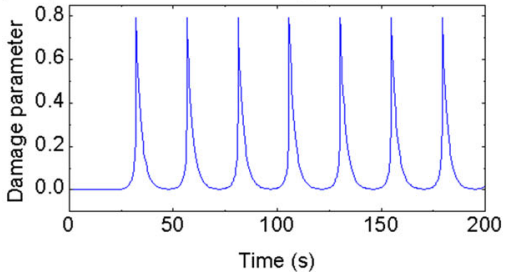

Fig. 15 Variations of shear stress, temperature rise and damage parameter in FM AZ31B at a wide range of cutting speeds: a1-a3 $V_{0}=10 \mathrm{~m} / \mathrm{s} ; \mathbf{b} \mathbf{1}-\mathbf{b} 3 V_{0}=10^{-1} \mathrm{~m} / \mathrm{s} ; \mathbf{c} 1-\mathbf{c} 3 V_{0}=10^{-2} \mathrm{~m} / \mathrm{s} ; \mathbf{d 1}-\mathbf{d} 3 V_{0}=10^{-5} \mathrm{~m} / \mathrm{s}$

$D$ which leads to stable limit cycles in the phase space of Fig. 16a, b. Compared with Fig. 16a, larger CEF in Fig. 16b leads to smaller limit cycles in the phase space. When CEF exceeds a certain value, e.g., CEF $\geq 0.44$ in the present experiments, the constraint is able to suppress the evolution of fracture in PSZ and enables the balance of the fracture-type damage convection, diffusion and source. As a result, steady-state shear stress $\tau$, temperature $T$ and damage parameter $D$ are achieved in Figs. 8c, d, 9 and 10c, d. Then, a homogeneous plastic flow occurs in PSZ, finally resulting in the continuous chip formation in Fig. 4c, d. With the help of the constraint, the static equilibrium of the fracture-type damage convection, diffusion and source indicates a limit point in the phase space (Fig. 16c, d). Compared with Fig. 16c, the larger CEF in Fig. 16d makes it faster to reach the limit point in the phase space.
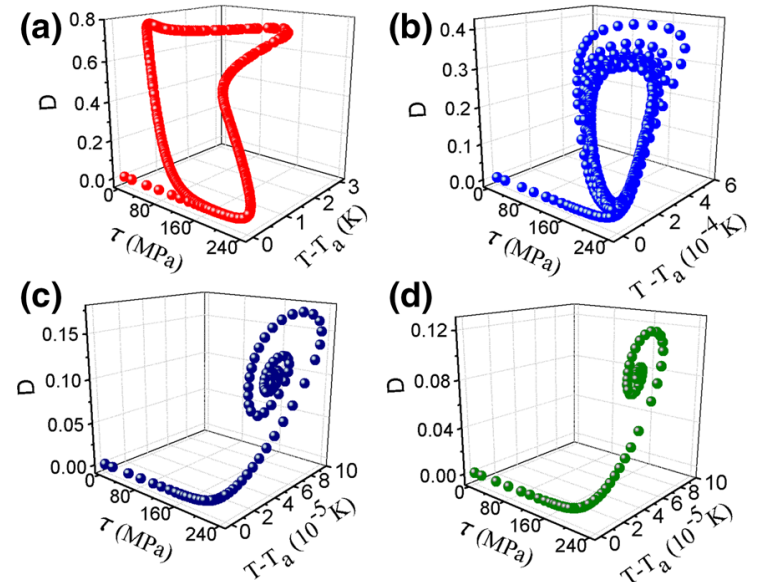

(d)

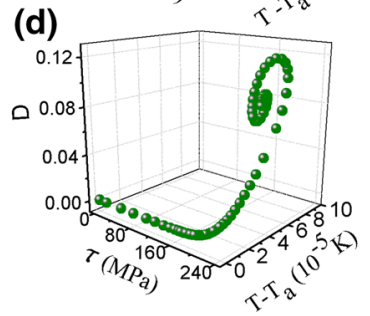

Fig. 16 Trajectories of shear stress $\tau$, temperature rise $T-T_{a}$ and damage parameter $D$ in low-speed extrusion machining AZ31B for different CEFs: $\mathbf{a} \mathrm{CEF}=0(\mathrm{FM})$; $\mathbf{b} \mathrm{CEF}=0.28$; c $\mathrm{CEF}=0.44 ; \mathbf{d} \mathrm{CEF}=0.52$ 
6.3 Criterion for Hopf bifurcation

Based on Eq. (8), the ordinary differential Eqs. (13)(16) are obtained by means of the dynamic parameters $\tau, V, T$ and $D$. Linear stability analysis of the governing system is carried out to reveal the effect of parameters $\left(V_{0}, \mathrm{CEF}\right)$ on Hopf bifurcation. According to the method of linear stability analysis [53-57], detailed processes of linear stability analysis are given out.

$$
\begin{aligned}
\frac{\mathrm{d} \tau}{\mathrm{d} t}= & \frac{E L_{1} \sin ^{2} \varphi}{t_{0}^{2}}\left(V_{0} \cos \varphi-V\right), \\
\frac{\mathrm{d} T}{\mathrm{~d} t}= & \frac{\beta \tau V}{\rho c h}+V_{0} \sin \varphi \frac{T_{a}-T}{h}+\frac{4 k}{\rho c} \frac{T_{a}-T}{h^{2}}, \\
\frac{\mathrm{d} D}{\mathrm{~d} t}= & (1-\beta)(1-D) \frac{\sqrt{3} \tau V}{G_{f}}-V_{0} \sin \varphi \frac{D}{h} \\
& -4 \tilde{\chi}_{D} \frac{D}{h^{2}}, \\
\tau= & g(V, T, D) .
\end{aligned}
$$

In order to simplify the governing Eqs. (13)-(16), the following dimensionless variables are introduced here:

$$
\left\{\begin{array}{l}
\hat{\tau}=\frac{\tau}{\tau_{y}}, \hat{T}=\frac{T-T_{a}}{T_{m}-T_{a}}, \hat{t}=\frac{E V_{0} t}{\tau_{y} t_{0}} \\
\hat{V}=\frac{V}{V_{0}}, \xi=\frac{\tau_{y}}{\rho c\left(T_{m}-T_{a}\right)} \\
F=\frac{\tau_{y} t_{0}}{E h}, H=\frac{V_{0} h}{\tilde{\chi}_{D}}, M=\frac{L_{1}}{t_{0}} \\
N=\frac{\sqrt{3}(1-\beta) \tau_{y}^{2} t_{0}}{E G_{f}}, P e=\frac{\rho c V_{0} h}{k}
\end{array}\right.
$$

Then, the non-dimensional governing system is the following form:

$$
\begin{aligned}
\frac{\mathrm{d} \hat{\tau}}{\mathrm{d} \hat{t}} & =M \sin ^{2} \varphi(\cos \varphi-\hat{V}), \\
\frac{\mathrm{d} \hat{T}}{\mathrm{~d} \hat{t}} & =\beta \xi F \hat{\tau} \hat{V}-F \sin \varphi \hat{T}-\frac{4 F \hat{T}}{P e},
\end{aligned}
$$

$$
\begin{aligned}
\frac{\mathrm{d} D}{\mathrm{~d} \hat{t}} & =(1-D) N \hat{\tau} \hat{V}-F \sin \varphi D-\frac{4 F D}{H}, \\
\hat{\tau} & =U(\hat{V}, \hat{T}, D) .
\end{aligned}
$$

For later use, let us introduce the following notations: $R=\frac{\partial U}{\partial \hat{V}}>0 \quad$ (strain rate hardening coefficient),

$P=-\frac{\partial U}{\partial \hat{T}}>0$ (thermal softening coefficient),

$O=-\frac{\partial U}{\partial D}>0 \quad$ (damage softening coefficient).

The governing equations have a steady state in the case of

$\frac{\mathrm{d} \hat{\tau}}{\mathrm{d} \hat{t}}=\frac{\mathrm{d} \hat{T}}{\mathrm{~d} \hat{t}}=\frac{\mathrm{d} D}{\mathrm{~d} \hat{t}}=0$.

The corresponding steady-state solutions are indicated as $\tau^{*}, T^{*}, D^{*}$ and $V^{*}$. There are two equilibrium points in the ODE model: One is a saddle node and the other is focus. Based on the physical background $\left(0 \leq T^{*} \leq 1\right.$ and $\left.0 \leq D^{*} \leq 1\right)$, the saddle node in the ODE model is not taken into consideration in the following discussion. Carrying out linear stability analysis of Eqs. (18)(21), that is

$$
\left(\begin{array}{l}
\hat{\tau} \\
\hat{T} \\
D \\
\hat{V}
\end{array}\right)=\left(\begin{array}{l}
\tau^{*} \\
T^{*} \\
D^{*} \\
V^{*}
\end{array}\right)+\left(\begin{array}{l}
\delta \hat{\tau} \\
\delta \hat{T} \\
\delta D \\
\delta \hat{V}
\end{array}\right) \exp (\lambda t) .
$$

Substituting Eq. (26) into Eqs. (18)-(21) and retaining the linear terms, the linearization governing system is given by:

$J \cdot\left(\begin{array}{c}\delta \hat{\tau} \\ \delta \hat{T} \\ \delta D \\ \delta \hat{V}\end{array}\right)=0$,

where

$$
J=\left[\begin{array}{cccc}
\lambda & 0 & 0 & M \sin ^{2} \varphi \\
\beta \xi F V^{*} & -\lambda-F \sin \varphi-\frac{4 F}{P e} & 0 & \beta \xi F \tau^{*} \\
N\left(1-D^{*}\right) V^{*} & 0 & -\lambda-N \tau^{*} V^{*}-F \sin \varphi-\frac{4 F}{H} & N\left(1-D^{*}\right) \tau^{*} \\
1 & P^{*} & O^{*} & -R^{*}
\end{array}\right] .
$$


$P^{*}, R^{*}$ and $O^{*}$ are defined by:

$\left\{\begin{array}{l}P^{*}=-\left.\frac{\partial U}{\partial \hat{T}}\right|_{\left(V^{*}, \tau^{*}, T^{*}, D^{*}\right)} \\ R^{*}=\left.\frac{\partial U}{\partial \hat{V}}\right|_{\left(V^{*}, \tau^{*}, T^{*}, D^{*}\right)} \\ O^{*}=-\left.\frac{\partial U}{\partial D}\right|_{\left(V^{*}, \tau^{*}, T^{*}, D^{*}\right)}\end{array}\right.$.

To have a non-trivial solution, the determinant of matrix $J$ must be equal to zero. This leads to a thirdorder eigenvalue equation:

$a_{1} \lambda^{3}+a_{2} \lambda^{2}+a_{3} \lambda+a_{4}=0$,

where the polynomial coefficients are

$$
\left\{\begin{aligned}
a_{1}= & R^{*} \\
a_{2}= & \frac{4 F R^{*}}{H}+\frac{4 F R^{*}}{P e}+2 F R^{*} \sin \varphi+M \sin ^{2} \varphi \\
& +N R^{*} V^{*} \tau^{*}-N O^{*}\left(1-D^{*}\right) \tau^{*}-F \beta \xi P^{*} \tau^{*} \\
a_{3}= & \frac{16 F^{2} R^{*}}{H P e}+\frac{4 F^{2} R^{*} \sin \varphi}{H}+\frac{4 F^{2} R^{*} \sin \varphi}{P e} \\
& +\frac{4 F M \sin ^{2} \varphi}{H}+\frac{4 F M \sin ^{2} \varphi}{P e}+F^{2} R^{*} \sin ^{2} \varphi \\
& +2 F M \sin ^{3} \varphi+M N O^{*} \sin ^{2} \varphi\left(1-D^{*}\right) V^{*} \\
& +F M P^{*} \sin ^{2} \varphi \beta \xi V^{*}+\frac{4 F N R^{*} V^{*} \tau^{*}}{P e} \\
& +F N R^{*} \sin \varphi V^{*} \tau^{*}+M N \sin ^{2} \varphi V^{*} \tau^{*} \\
& -\frac{4 F N O^{*}\left(1-D^{*}\right) \tau^{*}}{P e}-F N O^{*} \sin \varphi\left(1-D^{*}\right) \tau^{*} \\
& -\frac{4 F^{2} P^{*} \beta \xi \tau^{*}}{H}-F^{2} P^{*}{\sin \varphi \beta \xi \tau^{*}} \\
& -F N P^{*} \beta \xi V^{*}\left(\tau^{*}\right)^{2} \\
a_{4}= & \frac{16 F^{2} M \sin ^{2} \varphi}{H P e}+\frac{4 F^{2} M \sin ^{3} \varphi}{H}+\frac{4 F^{2} M \sin ^{3} \varphi}{P e} \\
& +F^{2} M \sin ^{4} \varphi+\frac{4 F M N O^{*} \sin ^{2} \varphi\left(1-D^{*}\right) V^{*}}{P e} \\
& +F M N O^{*} \sin ^{3} \varphi\left(1-D^{*}\right) V^{*} \\
& +\frac{4 F^{2} M P^{*} \sin ^{2} \varphi \beta \xi V^{*}}{H}+F^{2} M P^{*} \sin ^{3} \varphi \beta \xi V^{*} \\
& +\frac{4 F M N \sin ^{2} \varphi V^{*} \tau^{*}}{P e}+F M N \sin ^{3} \varphi V^{*} \tau^{*} \\
& +F M N P^{*} \sin ^{2} \varphi \beta \xi\left(V^{*}\right)^{2} \tau^{*}
\end{aligned}\right.
$$

The stability is determined by the real part of eigenvalue $\lambda$ : If an eigenvalue $\lambda$ exists with its real part $\operatorname{Re}(\lambda)>0$, the solution is unstable; otherwise, it is stable. Based on Routh-Hurwitz criterion [58], the stable conditions are given by

$$
\left\{\begin{array}{l}
a_{2}>0 \\
a_{3}>0 \\
a_{2} a_{3}>a_{1} a_{4}
\end{array} .\right.
$$

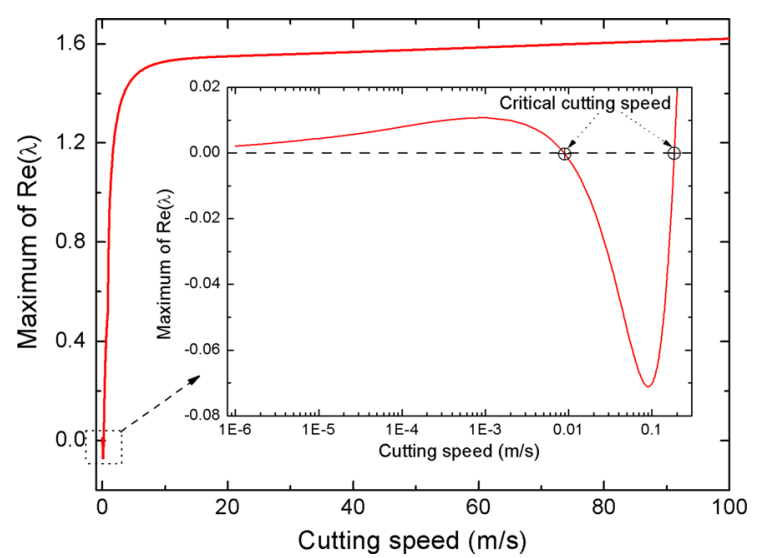

Fig. 17 Relationship between the maximum of $\operatorname{Re}(\lambda)$ and cutting speed for a given CEF

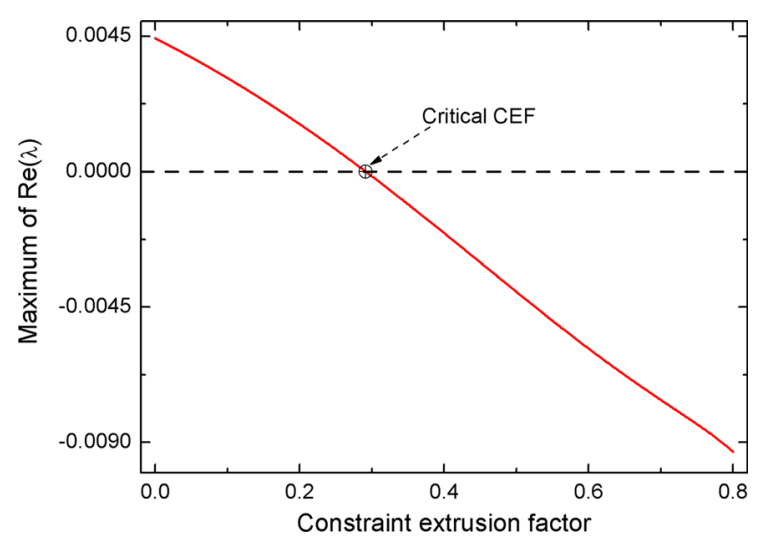

Fig. 18 Relationship between the maximum of $\operatorname{Re}(\lambda)$ and CEF for a given cutting speed

Based on Eq. (29), the relationship between the maximum of $\operatorname{Re}(\lambda)$ and cutting speed is calculated (see in Fig. 17). For a given CEF during machining AZ31B (e.g., $\mathrm{CEF}=0$ ), there are two critical cutting speeds $(0.01$ and $0.2 \mathrm{~m} / \mathrm{s})$. If the cutting speed is lower than $0.01 \mathrm{~m} / \mathrm{s}$, the real part of eigenvalue is greater than zero and then Hopf bifurcation gives rise to chip segmentation. If the cutting speed is higher than $0.2 \mathrm{~m} / \mathrm{s}$, the real part of eigenvalue is also greater than zero and then Hopf bifurcation gives rise to chip segmentation. When the cutting speed is between 0.01 and $0.2 \mathrm{~m} / \mathrm{s}$, the eigenvalue $\lambda$ cannot exist with its real part $\operatorname{Re}(\lambda)>0$; therefore, there is no Hopf bifurcation.

The relationship between the maximum of $\operatorname{Re}(\lambda)$ and CEF is shown in Fig. 18 by numerical solutions of Eq. (29). For a given cutting speed during machining AZ31B (e.g., $10 \mu \mathrm{m} / \mathrm{s})$, the maximum of $\operatorname{Re}(\lambda)$ 


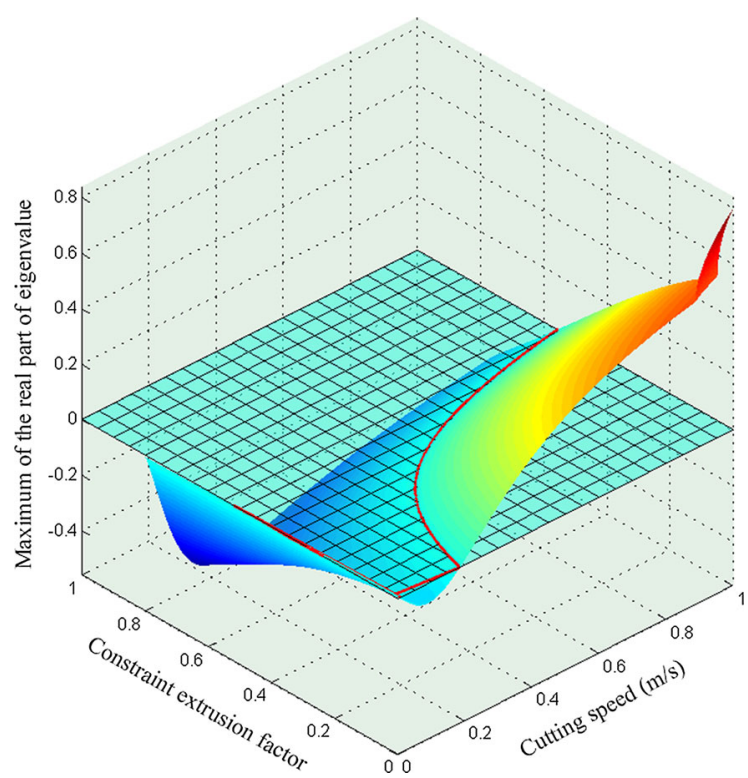

Fig. 19 Variation of the maximum of $\operatorname{Re}(\lambda)$ with cutting speed and CEF. (Color figure online)

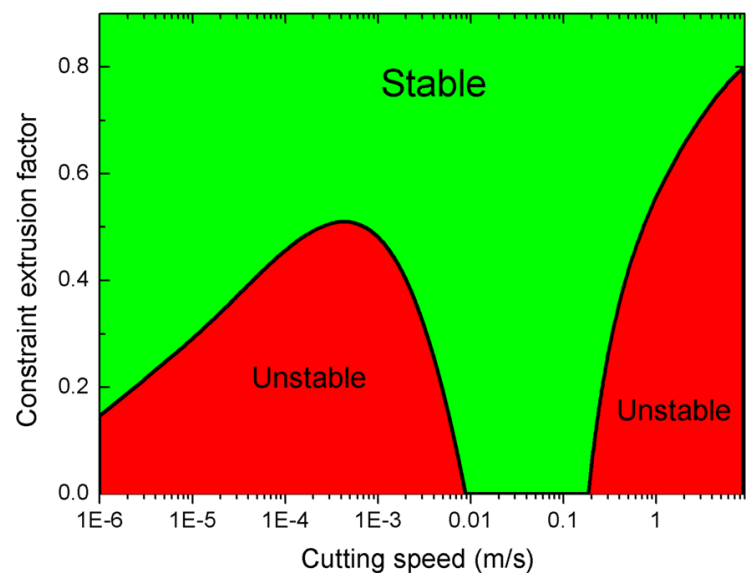

Fig. 20 Phase diagram of Hopf bifurcation in the parameter plane $\left(V_{0}, \mathrm{CEF}\right)$. (Color figure online)

decreases with the increasing CEF. When CEF exceeds a certain value (e.g., $C E F=0.29$ ), the eigenvalue $\lambda$ cannot exist with its real part $\operatorname{Re}(\lambda)>0$; otherwise, Hopf bifurcation gives rise to chip segmentation. The calculated results are in agreement with the experimental observations (see in Fig. 4).

Based on Eq. (29), the occurrence of Hopf bifurcation is analyzed by choosing either the cutting speed or the CEF as bifurcation parameters. The variation of the maximum of $\operatorname{Re}(\lambda)$ with cutting speed and CEF is illustrated in Fig. 19 by substituting the machining parameters of AZ31B into Eq. (29). The curved surface is divided into two parts by a red curve. The parameters $\left(V_{0}, \mathrm{CEF}\right)$ located on the upper part of the curved surface would lead to the occurrence of Hopf bifurcation; otherwise, there is no Hopf bifurcation during the formation of chips. Furthermore, Eq. (31) gives out the loci of Hopf bifurcation by choosing either the cutting speed or the CEF as bifurcation parameters. As shown in Fig. 20, the phase diagram of Hopf bifurcation is indicated in the parameter plane $\left(V_{0}, C E F\right)$. If the parameters $\left(V_{0}, \mathrm{CEF}\right)$ locate in the green region, the deformation in chips is stable; otherwise, Hopf bifurcation happens during the formation of chips (e.g., the red region in Fig. 20).

\section{Concluding remarks}

In summary, using low-speed extrusion machining technique, the systematic extrusion machining experiments of magnesium alloy AZ31B and aluminum alloy 7075-T6 were conducted for different constraint extrusion factors (CEFs) at the cutting speed of $10^{-5} \mathrm{~m} / \mathrm{s}$. The microscopic observations of chips reveal that the chip is segmented during free machining (FM) at the relatively low cutting speed, which is different from the existing viewpoint that the chip transforms from serrated to continuous with the decreasing cutting speed. The comparison of microstructures for different CEFs shows the transition from fracture-type segmented deformation to homogeneous sheet deformation with the increasing CEF in low-speed extrusion machining. The existing thermal-softening-based model is invalid to explain the achieved experimental results. Based on the low-speed extrusion machining experimental observations, we present a nonlinear dynamic model for extrusion machining, taking into account fracture-type deformation, thermal-softeningtype deformation, effects of extrusion constraint, material convection and momentum diffusion. Compared with the existing thermal-softening-based model, the presented theoretical model is more rational to explain the experimental results; therefore, the presented theoretical model is effective to characterize the process of chip formation in extrusion machining.

According to the numerical calculations of the presented model, we reveal the mechanism underlying in the chips during low-speed extrusion machining. For small CEF, the symmetry breaking of the fracture- 
type damage convection, diffusion and source governs the segmented chips in low-speed extrusion machining, which can be understood as the manifestation of a self-sustained stable limit-cycle bifurcation phenomenon. As for large CEF, with the help of the suppression of fracture from the constraint, the balance of the fracture type damage convection, diffusion and source results in the homogeneous chips corresponding to a limit point at mathematics. During low-speed extrusion machining, we should focus on fracture-type deformation and thermal-softening-type deformation is less important; however, thermal-softening-type deformation is the primary deformation mode and fracturetype deformation is negligible for high-speed machining.

Acknowledgements This work has been supported by the National Natural Science Foundation of China (Grant Nos. 11132011 and 11602236), Fundamental Research Funds for the Central Universities (Grant No. FRF-TP-15-101A1), the Opening Fund of State Key Laboratory of Nonlinear Mechanics, China Postdoctoral Science Foundation (Grant No. 2016M591066), the National Basic Research Program of China (Grant No. 2012CB937500) and the CAS/SAFEA International Partnership Program for Creative Research Teams. We are very grateful to the anonymous reviewers for their helpful comments, which have improved our manuscript significantly.

\section{References}

1. Shaw, M.C.: Metal Cutting Principles, 2nd edn. Oxford University Press, Oxford (2005)

2. Brown, T.L., Swaminathan, S., Chandrasekar, S., Compton, W.D., King, A.H., Trumble, K.P.: Low-cost manufacturing process for nanostructured metals and alloys. J. Mater. Res. 17(10), 2484-2488 (2002). doi:10.1557/jmr.2002.0362

3. Cai, S.L., Chen, Y., Ye, G.G., Jiang, M.Q., Wang, H.Y., Dai, L.H.: Characterization of the deformation field in largestrain extrusion machining. J. Mater. Process. Technol. 216, 48-58 (2015). doi:10.1016/j.jmatprotec.2014.08.022

4. Liu, Y., Cai, S., Dai, L.: A new method for grain refinement in magnesium alloy: high speed extrusion machining. Mater. Sci. Eng. A 651, 878-885 (2016). doi:10.1016/j.msea.2015. 11.046

5. Otto, A., Radons, G.: The influence of tangential and torsional vibrations on the stability lobes in metal cutting. Nonlinear Dyn. 82(4), 1989-2000 (2015). doi:10.1007/ s11071-015-2293-9

6. Deng, W.J., Xia, W., Li, C., Tang, Y.: Formation of ultrafine grained materials by machining and the characteristics of the deformation fields. J. Mater. Process. Technol. 209(9), 4521-4526 (2009). doi:10.1016/j.jmatprotec.2008.10.043

7. Recht, R.F.: Catastrophic thermoplastic shear. J. Appl. Mech. 31(2), 189-193 (1964)
8. Semiatin, S.L., Rao, S.B.: Shear localization during metal cutting. Mater. Sci. Eng. 61(2), 185-192 (1983). doi:10. 1016/0025-5416(83)90200-8

9. Molinari, A., Dudzinski, D.: Stationary shear band in highspeed machining. C. R. Acad. Sci. Ser. 315(4), 399-405 (1992)

10. Burns, T.J., Davies, M.A.: Nonlinear dynamics model for chip segmentation in machining. Phys. Rev. Lett. 79(3), 447-450 (1997). doi:10.1103/PhysRevLett.79.447

11. Burns, T.J., Davies, M.A.: On repeated adiabatic shear band formation during high-speed machining. Int. J. Plast. 18(4), 487-506 (2002). doi:10.1016/s0749-6419(01)00006-7

12. Huang, J., Aifantis, E.C.: A note on the problem of shear localization during chip formation in orthogonal machining. J. Mater. Eng. Perform. 6(1), 25-26 (1997). doi:10.1007/ s11665-997-0027-y

13. Huang, J., Kalaitzidou, K., Sutherland, J.W., Aifantis, E.C.: Validation of a predictive model for adiabatic shear band formation in chips produced via orthogonal machining. J. Mech. Behav. Mater. 18(4), 243-263 (2007)

14. Molinari, A., Soldani, X., Miguélez, M.H.: Adiabatic shear banding and scaling laws in chip formation with application to cutting of Ti-6A1-4V. J. Mech. Phys. Solids 61(11), 23312359 (2013). doi:10.1016/j.jmps.2013.05.006

15. Hanna, N.H., Tobias, S.A.: A theory of nonlinear regenerative chatter. ASME J. Eng. Ind. 96, 247-255 (1974)

16. Shi, H.M., Tobias, S.A.: Theory of finite amplitude machine tool instability. Int. J. Mach. Tool Des. Res. 24, 45-69(1984)

17. Nayfeh, A.H., Chin, C.M., Pratt, J.R.: Perturbation methods in nonlinear dynamics: applications to machining dynamics. J. Manuf. Sci. Eng. 119, 485-493 (1997)

18. Pratt, J.R., Nayfeh, A.H.: Design and modeling for chatter control. Nonlinear Dyn. 19, 49-69 (1999)

19. Kalmár-Nagy, T., Stépán, G., Moon, F.C.: Subcritical Hopf bifurcation in the delay equation model for machine tool vibrations. Nonlinear Dyn. 26(2), 121-142 (2001). doi:10. 1023/a:1012990608060

20. Nayfeh, A.H., Nayfeh, N.A.: Analysis of the cutting tool on a lathe. Nonlinear Dyn. 63(3), 395-416 (2010). doi:10. 1007/s11071-010-9811-6

21. Ren, Q., Baron, L., Balazinski, M.: Fuzzy identification of cutting acoustic emission with extended subtractive cluster analysis. Nonlinear Dyn. 67(4), 2599-2608 (2011). doi:10. 1007/s11071-011-0173-5

22. Moradi, H., Movahhedy, M.R., Vossoughi, G.: Bifurcation analysis of milling process with tool wear and process damping: regenerative chatter with primary resonance. Nonlinear Dyn. 70(1), 481-509 (2012). doi:10.1007/ s11071-012-0470-7

23. Litak, G., Schubert, S., Radons, G.: Nonlinear dynamics of a regenerative cutting process. Nonlinear Dyn. 69(3), 1255 1262 (2012). doi:10.1007/s11071-012-0344-z

24. Elias, J., Namboothiri, V.N.N.: Cross-recurrence plot quantification analysis of input and output signals for the detection of chatter in turning. Nonlinear Dyn. 76(1), 255-261 (2013). doi:10.1007/s11071-013-1124-0

25. Molnár, T.G., Insperger, T., Stépán, G.: State-dependent distributed-delay model of orthogonal cutting. Nonlinear Dyn. (2015). doi:10.1007/s11071-015-2559-2

26. Ye, G.G., Xue, S.F., Jiang, M.Q., Tong, X.H., Dai, L.H.: Modeling periodic adiabatic shear band evolution during 
high speed machining Ti-6Al-4V alloy. Int. J. Plast. 40, 39-55 (2013). doi:10.1016/j.ijplas.2012.07.001

27. Molinari, A., Musquar, C., Sutter, G.: Adiabatic shear banding in high speed machining of Ti-6Al-4V: experiments and modeling. Int. J. Plast. 18(4), 443-459 (2002). doi:10.1016/ s0749-6419(01)00003-1

28. Efe, M., Moscoso, W., Trumble, K.P., Dale Compton, W., Chandrasekar, S.: Mechanics of LSEM and application to deformation processing of magnesium alloys. Acta Mater. 60(5), 2031-2042 (2012). doi:10.1016/j.actamat.2012.01. 018

29. Shankar, M.R., Rao, B.C., Lee, S., Chandrasekar, S., King, A.H., Compton, W.D.: Severe plastic deformation (SPD) of titanium at near-ambient temperature. Acta Mater. 54(14), 3691-3700 (2006). doi:10.1016/j.actamat.2006.03.056

30. Sheikh-Ahmad, J.Y., Quarless, V., Bailey, J.A.: On the role of microcracks on flow instability in low speed machining of CP titanium. Mach. Sci. Technol. 8(3), 415-430 (2004). doi:10.1081/lmst-200039867

31. Cai, S.L., Dai, L.H.: Suppression of repeated adiabatic shear banding by dynamic large strain extrusion machining. J. Mech. Phys. Solids 73, 84-102 (2014). doi:10.1016/j.jmps. 2014.09.004

32. De Chiffre, L.: Extrusion-cutting. Int. J. Mach. Tool Des. Res. 16(2), 137-144 (1976). doi:10.1016/ 0020-7357(76)90032-9

33. Oxley, P.L.B.: Mechanics of Machining: An Analytical Approach to Assessing Machinability. Wiley, New York (1989)

34. Wallace, D.C.: Irreversible thermodynamics of flow in solids. Phys. Rev. B 22, 1477-1786 (1980)

35. Taylor, G.I., Quinney, H.: The plastic distortion of metals. Philos. Trans. R. Soc. Lond. A 230, 323-362 (1931)

36. Wright, T.W., Walter, J.W.: The asymptotic structure of an adiabatic shear band in antiplane motion. J. Mech. Phys. Solids 44(1), 77-97 (1996). doi:10.1016/ 0022-5096(95)00066-6

37. Bai, Y.L.: Thermo-plastic instability in simple shear. J. Mech. Phys. Solids 30(4), 195-207 (1982). doi:10.1016/ 0022-5096(82)90029-1

38. Molinari, A.: Collective behavior and spacing of adiabatic shear bands. J. Mech. Phys. Solids 45(9), 1551-1575 (1997). doi:10.1016/s0022-5096(97)00012-4

39. Jiang, M.Q., Dai, L.H.: On the origin of shear banding instability in metallic glasses. J. Mech. Phys. Solids 57(8), 12671292 (2009). doi:10.1016/j.jmps.2009.04.008

40. Dolinski, M., Rittel, D.: Experiments and modeling of ballistic penetration using an energy failure criterion. J. Mech. Phys. Solids 83, 1-18 (2015). doi:10.1016/j.jmps.2015.06. 004

41. Dolinski, M., Rittel, D., Dorogoy, A.: Modeling adiabatic shear failure from energy considerations. J. Mech. Phys. Solids 58(11), 1759-1775 (2010). doi:10.1016/j.jmps.2010. 08.007

42. Hillerborg, A., Modeer, M., Petersson, P.E.: Analysis of crack formation and crack growth in concrete by means of fracture mechanics and finite elements. Cem. Concr. Res. 6, 773-782 (1976)
43. Zhang, Y.C., Mabrouki, T., Nelias, D., Gong, Y.D.: Chip formation in orthogonal cutting considering interface limiting shear stress and damage evolution based on fracture energy approach. Finite Elem. Anal. Des. 47(7), 850-863 (2011). doi:10.1016/j.finel.2011.02.016

44. Merchant, M.E.: Mechanics of the metal cutting process. II. Plasticity conditions in orthogonal cutting. J. Appl. Phys. 16(6), 318-324 (1945)

45. Merchant, M.E.: Mechanics of the metal cutting process. I. Orthogonal cutting and a type 2 chip. J. Appl. Phys. 16(5), 267-275 (1945)

46. Sun, S., Brandt, M., Dargusch, M.S.: Characteristics of cutting forces and chip formation in machining of titanium alloys. Int. J. Mach. Tools Manuf. 49(7-8), 561-568 (2009). doi:10.1016/j.ijmachtools.2009.02.008

47. Zhou, L., Peng, F.Y., Yan, R., Yao, P.F., Yang, C.C., Li, B.: Analytical modeling and experimental validation of micro end-milling cutting forces considering edge radius and material strengthening effects. Int. J. Mach. Tools Manuf. 97, 29-41 (2015). doi:10.1016/j.ijmachtools.2015.07.001

48. Sutter, G., List, G.: Very high speed cutting of Ti-6Al-4V titanium alloy: change in morphology and mechanism of chip formation. Int. J. Mach. Tools Manuf. 66, 37-43 (2013). doi:10.1016/j.ijmachtools.2012.11.004

49. Davies, M.A., Burns, T.J.: Thermomechanical oscillations in material flow during high-speed machining. Philos. Trans. R. Soc. Lond. A Math. Phys. Eng. Sci. 359(1781), 821-846 (2001)

50. Ye, G.G., Chen, Y., Xue, S.F., Dai, L.H.: Critical cutting speed for onset of serrated chip flow in high speed machining. Int. J. Mach. Tools Manuf. 86, 18-33 (2014). doi:10. 1016/j.ijmachtools.2014.06.006

51. Özel, T., Altan, T.: Determination of workpiece flow stress and friction at the chip-tool contact for high-speed cutting. Int. J. Mach. Tools Manuf. 40(1), 133-152 (2000). doi:10. 1016/s0890-6955(99)00051-6

52. Komanduri, R.: Some clarifications on the mechanics of chip formation when machining titanium alloys. Wear 76(1), 1534 (1982). doi:10.1016/0043-1648(82)90113-2

53. Wang, Z.H., Hu, H.Y.: Robust stability test for dynamic systems with short delays by using padé approximation. Nonlinear Dyn. 18, 275-287 (1999)

54. Wang, Z.H., Hu, H.Y.: Dimensional reduction for nonlinear time-delayed systems composed of stiff and soft substructures. Nonlinear Dyn. 25, 317-331 (2001)

55. Zhan, Z.Q., Zhu, J., Li, W.: Stability and bifurcation analysis in a FAST TCP model with feedback delay. Nonlinear Dyn. 70(1), 255-267 (2012)

56. Li, Y., Xu, D.: Chaotification of quasi-zero-stiffness system with time delay control. Nonlinear Dyn. 86, 353-368 (2016)

57. Zhang, X.J., Xiong, C.H., Ding, Y., Ding, H.: Updated numerical integration method for stability calculation of Mathieu equation with various time delays. Nonlinear Dyn. (2016). doi:10.1007/s11071-016-3096-3

58. Korn, G.A., Korn, T.M.: Mathermatical Handbook for Scientists and Engineers. McGraw-Hill, New York (1961) 